\title{
4. OLIGOCENE CALCAREOUS-NANNOFOSSIL BIOSTRATIGRAPHY FROM LEG 101, SITE 628, LITTLE BAHAMA BANK SLOPE 1
}

\author{
Michael J. Moran² and David K. Watkins²
}

\begin{abstract}
Drilling at Hole 628A, Leg 101 of the Ocean Drilling Program, recovered a thick, relatively complete Oligocene section of nannofossil-foraminifer chalk and ooze. Sediments from Cores 101-628A-16H through 101-628A-29X were examined using the light microscope to provide information about biostratigraphy of calcareous nannofossils. Several authors noted a problem in distinguishing the first appearance of species within the Sphenolithus predistentus-S. ciperoensis lineage of the mid-Oligocene, particularly Sphenolithus ciperoensis, because of the gradual evolution of its members. Successful separation of species in this lineage is critical for accurately dating relative age of mid- and upperOligocene nannofossil sediments. Length and taper of the apical spine, along with width of the proximal shield, were reported by various authors as the best criteria for separating $S$. ciperoensis from Sphenolithus distentus in light microscopy. We find that the "extinction" line characteristics of the proximal shield, as originally proposed, are the best criteria for separation in light microscopy. These criteria provide results that correlate well with dating using other microfossils. Scanning electron microscopy (SEM) study shows that this feature directly results from the widening of the proximal shield during evolution from $S$. predistentus to $S$. ciperoensis. Transitional forms between $S$. distentus and $S$. ciperoensis show features of both species. The last appearance datums (LAD) of Lanternithus minutus and Helicopontosphaera compacta were found to correspond with the first appearance datum (FAD) of S. ciperoensis in Hole 628A. The LAD of $H$. compacta has a high confidence rating for reliability as an alternative datum to the FAD of $S$. ciperoensis. The usefulness of $L$. minutus to approximate this datum is much less. Other potentially useful Oligocene nannofossil datums (with varying degrees of usefulness) also are given.
\end{abstract}

\section{INTRODUCTION}

Drilling at Hole 628A, Leg 101 of the Ocean Drilling Program, recovered a thick, relatively complete section of Oligocene nannofossil-foraminifer chalk and ooze. This section allowed us to examine the Oligocene nannofossil biostratigraphy in detail, particularly the mid-Oligocene, where a number of problems have arisen repeatedly regarding the Spheneolithus predistentus-Sphenolithus ciperoensis nannofossil lineage. Several authors noted a problem when working with the mid-Oligocene sphenoliths (e.g., Roth et al., 1971a, 1971b; Haq, 1972; Lang and Watkins, 1984) because of the slow evolution of this lineage. A major problem is to distinguish the FAD of Sphenolithus ciperoensis because of its gradual evolution from Sphenolithus distentus. Recognizing the FAD of members of this lineage is important for placing mid-Oligocene pelagic sediments containing nannofossils in the proper zone and, thus, for accurately dating relative age. Hole $628 \mathrm{~A}$ contained a thick section in the S. ciperoensis Zone (Cores 101-628A-16H through 101$628 \mathrm{~A}-24 \mathrm{X}$ ) that allowed us to study the $S$. distentus-S. ciperoensis transition in detail. We focused special attention on these two species in hopes of clarifying distinguishing characteristics for better taxonomic separation. In addition, the last appearances of other nannofossil species were considered as alternatives to the sphenolith datums.

Hole $628 \mathrm{~A}$ was located at $27^{\circ} 31.85^{\prime} \mathrm{N}, 78^{\circ} 18.95^{\prime} \mathrm{W}$, north of the Little Bahama Bank at the bank toe-of-slope in $966 \mathrm{~m}$ of water (Fig. 1). Holes 628A, 627, 629, and 630 were part of a transect of the Little Bahama Bank. Total penetration at the site was $298.4 \mathrm{~m}$. Sediments ranging from upper Paleocene through the Holocene were recovered. Shipboard nannofossil and fora-

\footnotetext{
${ }^{1}$ Austin, J. A., Jr., Schlager, W., et al., 1988. Proc. ODP, Sci. Results, 101: College Station, TX (Ocean Drilling Program).

2 Department of Geology, 330 Bessey Hall, University of Nebraska, Lincoln, NE 68588-0340.
}

minifer biostratigraphy indicated that Cores $101-628 \mathrm{~A}-16 \mathrm{H}$ through 101-628A-29X spanned the upper Oligocene to upper Eocene. Recovery from this interval was highly variable and ranged from $100+\%$ in Cores $101-628 \mathrm{~A}-16 \mathrm{H}$ and $101-628 \mathrm{~A}$ $22 \mathrm{X}$ to $0 \%$ in Core $101-628 \mathrm{~A}-20 \mathrm{H}$ (only a core-catcher sample was available for analysis). The average recovery through this interval was 69\% (Austin, Schlager, et al., 1986). Cores 101-628A$25 \mathrm{X}$ through 101-628A-29X had a recovery of less than $50 \%$.

Lithologically, Cores 101-628A-16H through 101-628A-23X and $101-628 \mathrm{~A}-27 \mathrm{X}$ through $101-628 \mathrm{~A}-29 \mathrm{X}$ consisted predominantly of foraminifer-nannofossil chalk and ooze. Cores 101628A-24X through 101-628A-26X consisted predominantly of varying proportions of nannofossil-foraminifer chalk and ooze and lithified or partially lithified packstones and floatstones. Bioturbation was indicated in all cores in varying amounts (Austin, Schlager, et al., 1986).

\section{METHODS AND PROCEDURES}

Calcareous-nannofossil assemblages from samples of Cores 101-628A$16 \mathrm{H}$ through 101-628A-29X were examined using the light microscope to provide information about biostratigraphy. Results from the examination of smear slides prepared from raw-sediment samples are presented in Table 1. This table shows the relative abundance of each nannofossil taxon, its distribution, and an estimate of its preservational state in each core sample examined. Estimation of abundance and preservation of individual nannofossil species follows Hay (1970), as modified by Watkins and Bowdler (1984). Abundances for individual nannofossil taxa in Table 1 are as follows: $A$, abundant (1-10 specimens per field of view at $1500 \times) ; C$, common (1 specimen per 2-10 fields of view at $1500 \times) ; F$, few (1 specimen per 11-100 fields of view at $1500 \times)$; $R$, rare $(1 \mathrm{speci}$ men per 101-1000 fields of view at $1500 \times$ ); ?, questionable presence of the species.

Our method for estimating the abundance of nannofossils as a sedimentary component was taken from Watkins and Bowdler (1984). All except one sample (Sample 101-628A-23X-2, 95-100 cm) contained abundant nannofossils ( $>15 \%$ ). In this sample, nannofossils are diluted by a large component of quartz silt. Several samples of periplatform ooze within the section contained abundant micrite debris, while still having abundant (more than 15\%) nannofossils. These samples are denoted by the letter " $P$ " in the abundance column of Table 1. 


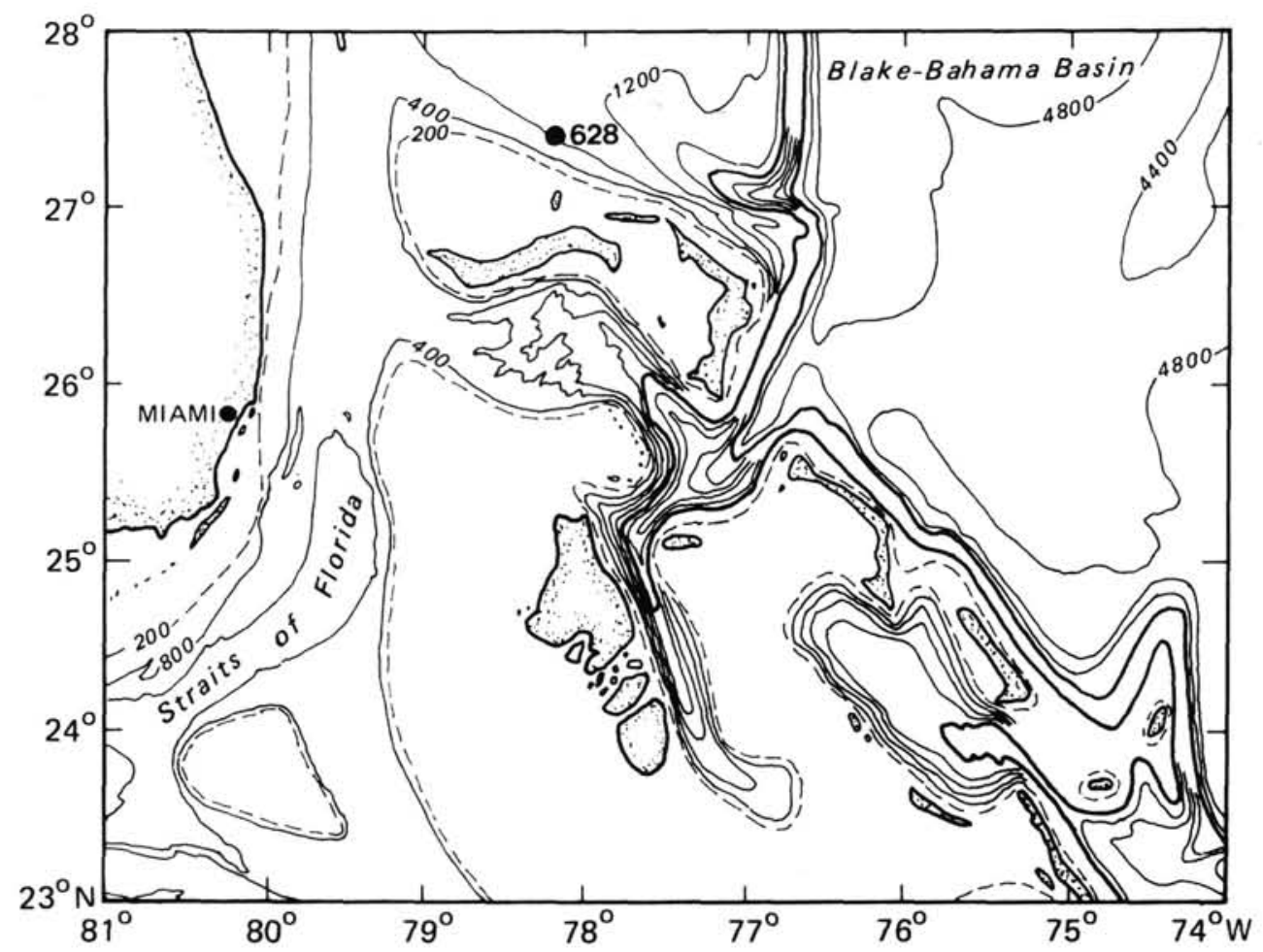

Figure 1. Location of Site 628, Leg 101. Bathymetry shown in meters. Adapted from Austin, Schlager, et al. (1986, front end-paper map).

Preservation of nannofossils in any given sample varies greatly. An "average" state of preservation was assigned to each sample, based on the following method, which follows Watkins and Bowdler (1984), G (good) = most specimens exhibit little or no secondary alteration; $\mathbf{M}$ (moderate $=$ specimens exhibit the effects of secondary alteration caused by etching and/or overgrowth (identification of species is not impaired); $\mathrm{P}$ (poor) $=$ specimens exhibit profound effects of secondary alteration from etching and/or overgrowth (identification of species is impaired but possible). In general, preservation is good to moderate throughout, with only one sample (101-628A-23X-2, 95-100 cm) exhibiting poor preservation of nannofossils.

Biostratigraphic zonation of the samples follows Bukry (1973a, 1975 ) and Okada and Bukry (1980). Their scheme was used because it gives the highest resolution for the Oligocene and is more applicable to tropical regions. The good recovery in many of the cores allowed us to recognize most of the Oligocene zones and subzones of Okada and Bukry (1980). Zonal and subzonal names were modified to conform with the taxonomy used here.

\section{RESULTS}

Results about the relative abundance, preservation, and distribution of nannofossils from examination of Hole 628A core samples are presented in Table 1. At the top of the studied interval, Samples 101-628A-16H-1, 80-81 cm through 101-628A-23X, $\mathrm{CC}$ are assigned to the Dictyococcites bisectus Subzone (CP19b) of the Sphenolithus ciperoensis Zone (CP19). This subzone is clearly delineated by the last appearance of $S$. distentus, which occurs in Sample 101-628A-24X-1, 80-81 cm. Typical species for this subzone include Coccolithus eopelagicus, Coccolithus pelagicus, Coronocyclus nitescens, Cyclicargolithus abisectus, Cyclicargolithus floridanus, Discoaster deflandrei, Ericsonia fenestrata, Helicopontosphaera euphratis, $H$. recta, Dictyococcites bisectus, Sphenolithus ciperoensis, and S. moriformis. No important datums were observed in this subzone.

Sample 101-628A-24X-1, 80-81 cm, is assigned to the Cyclicargolithus floridanus Subzone (CP19a) of the Sphenolithus ciperoensis Zone (CP19). This subzone was defined by Bukry
(1975) on the basis of the co-occurrence of S. ciperoensis and $S$. distentus. Sphenolithus distentus is present only in low abundance levels in this sample. The thinness of this subzone and the underlying Sphenolithus distentus Zone indicates that a hiatus may be present in this part of the section. Typical nannofossil species are the same as for the overlying subzone, with the addition of $S$. distentus. The last appearances of Helicopontosphaera compacta and Lanternithus minutus occur in Section 101-628A$24 \mathrm{H}, \mathrm{CC}$, which corresponds to the first appearance of $S$. ciperoensis at the base of the subzone. The last appearance of $H$. compacta may be a useful datum for approximating the first appearance of S. ciperoensis, which is discussed later. The LAD of L. minutus is less useful for approximating this datum.

Although the Cyclicargolithus floridanus Subzone is thin, the boundary between it and the underlying Sphenolithus distentus Zone (CP18) is difficult to delineate in this section. The original criteria for the Sphenolithus distentus Zone was the stratigraphic interval from the FAD of $S$. distentus to the first evolutionary appearance (FAD) of $S$. ciperoensis (Bukry, 1973a). However, previous workers noted a problem with the gradualistic nature in the evolution of the sphenoliths during the Oligocene, making the distinction of the FAD of $S$. ciperoensis difficult (Roth et al., 1971a, 1971b; Haq, 1972; Lang and Watkins, 1984). This problem also was encountered in the Oligocene section from the northern slope of Little Bahama Bank with forms that are transitional between $S$. distentus and $S$. ciperoensis occurring to the top of CP19. This caused confusion regarding the LAD of $S$. distentus and the exact taxonomic criteria for separating these species. A method for resolving this problem is discussed later. Using this methodology, we were able to make the following biostratigraphic subdivision of the section: Section 101-628A$24 \mathrm{X}$, CC through Sample 101-628A-25X-2, 80-81 cm, are assigned to the $S$. distentus Zone (CP18). The base of the zone is clearly marked by the first appearance of $S$. distentus, while the top corresponds to the first appearance of $S$. ciperoensis. The taxonomic composition of assemblages in this interval is similar 
to those in the overlying zone, with the addition of $H$. compacta and $L$. minutus.

Although this zone is thin $(\approx 3 \mathrm{~m})$ at this site, several important nannofossil datums can be recognized in it. The first common appearance of Cyclicargolithus abisectus occurs in Sample 101-628A-25X-1, 80-82 cm. Discoaster tani becomes extinct and $D$. adamanteus first appears near the FAD of $S$. distentus in Sample 101-628A-25X-2, 80-81 cm. Sphenolithus pseudoradians also become extinct in Sample 101-628A-25X-2, 80-81 cm. In some areas, the LAD of D. tani and the FAD of D. adamanteus might be useful for approximating the first appearance of $S$. distentus, as explained later.

The last appearance of $S$. predistentus occurs in Section 101628A-24X, CC, slightly before the last appearance of $S$. distentus in Sample 101-628A-24X-1, 80-81 cm. Perch-Nielsen (1985) stated that it is common for $S$. predistentus to disappear before the last appearance of $S$. distentus. Specimens of $S$. predistentus occur as high as Sample 101-628A-21H-3, 80-81 cm, in this section.

The thinness of the Sphenolithus distentus Zone, along with the disappearance of $S$. pseudoradians and several other nannofossils at the base of the zone, might indicate that part of this zone is missing. This idea gains credence because Lang and Watkins (1984) noticed a gradual change from $S$. predistentus to $S$. distentus in Oligocene material from DSDP Leg 77 . This change also was observed by Moran (in Watkins et al., this volume) in material from Site 540 , Leg 77 , which suggests that a gradual change exists between these two species, as between $S$. distentus and $S$. ciperoensis. This gradual change was not observed in material from Hole 628A because $S$. distentus appears suddenly. No transitional forms between $S$. distentus and $S$. predistentus were found. The lack of expression of a gradual transition between these two species may result from missing material because of an unconformity.

Section 101-628A-25X, CC through Sample 101-628A-27X-2, $80-81 \mathrm{~cm}$, are assigned to the Sphenolithus predistentus Zone (CP17), which approximates the transition from upper to lower Oligocene. This zone is defined as the stratigraphic interval between the FAD of $S$. distentus to the LAD of Reticulofenestra hillae and R. umbilica (Bukry, 1973a). A typical assemblage of this zone from Hole 628A includes Bramletteius serraculoides (few), Calcidiscus protoannula, Coccolithus eopelagicus, Coccolithus pelagicus, Coronocyclus nitescens, Cyclicargolithus floridanus, Discoaster deflandrei, D. tani, Helicopontosphaera compacta, Dictyococcites bisectus, Sphenolithus moriformis, $S$. predistentus, and $S$. pseudoradians. The LAD of Bramletteius serraculoides occurs in Sample 101-628A-27X-1, 80-81 cm, near the base of the zone. Chiasmolithus titus also becomes extinct near the base of the zone in Sample 101-628A-27X-1, 80-81 cm. Cyclicargolithus abisectus first appears (FAD) in Section 101628A-26X, CC. A few specimens of Helicopontosphaera reticulata and Reticulofenestra umbilica appear in Samples 101-628A$27 \mathrm{X}-2,80-81 \mathrm{~cm}$, and 101-628A-27X-1, 80-81 cm. A few Discoaster saipanensis also appear in these samples. Sparseness and poor preservation suggest that these specimens were reworked.

The lowermost zone, Helicopontosphaera reticulata (CP16), consists of these subzones (Fig. 2). The uppermost, Reticulofenestra hillae Subzone (CP16c), is separated from the underlying Ericsonia formosa Subzone (CP16b) by the LAD of Ericsonia formosa. This datum occurs between Sample 101-628A-28X-1, $80-81 \mathrm{~cm}$, and Sample 101-628A-27X-2, 80-81 cm. As a result, Section 101-628A-27X, CC is assigned to the $R$. hillae Subzone (CP16c). As this subzone is found only in a core-catcher sample, we infer that the section is very thin at best and that an unconformity may exist here.

Sample 101-628A-28X-1, 80-81 cm, is assigned to the $E$. formosa Subzone (CP16b). Samples 101-628A-28X-2, 80-81 cm, through 101-628A-29X-2, 47-48 cm, tentatively are assigned to the Ericsonia subdisticha Subzone (CP16a). Originally, Roth and Hay (1967) defined this zone as the interval from the LAD of Discoaster barbadiensis to the LAD of Cyclicoccolithus margaritae. Roth et al. (1971a) later refined the zone to include the interval from the LAD of $D$. barbadiensis to the LAD of Cyclicoccolithina formosa (= Ericsonia formosa). Bukry (1973a) defined this subzone on the basis of an acme of Ericsonia subdisticha that can be distinguished in some areas. All three authors listed Isthmolithus recurvus as an assemblage species, and Bukry (1973a) noted that the LAD of $I$. recurvus occurs in the overlying $E$. formosa Subzone. Although $E$. subdisticha is common throughout the material in question, we could differentiate no acme. Nevertheless, the samples are assigned to this subzone on the basis of the presence of $I$. recurvus and assemblages that are consistent with this subzonal assignment.

Assemblages for the $H$. reticulata Zone include Bramletteius serraculoides, Calcidiscus protoannula, Coccolithus eopelagicus, Coccolithus pelagicus, Ericsonia formosa, Ericsonia subdisticha, Coronocyclus nitescens, Cyclicargolithus floridanus, Discoaster deflandrei, D. tani, D. nodifer, Hayella situliformis, Helicopontosphaera compacta, Isthmolithus recurvus (basal subzone), Dictyococcites bisectus, $R$. hillae, $R$. umbilica, Sphenolithus moriformis, $S$. predistentus (few), and $S$. pseudoradians. Numerous specimens of the genus Pemma occur in the lowermost part of the subzone (Sample 101-628A-29X-2, 47-48 cm) but decrease in abundance until they disappear above Sample $101-628-28 X-2,80-81 \mathrm{~cm}$. The majority of these braarudosphaerids occur as fragments. In general, braarudosphaerids are most common in samples near the Eocene/Oligocene boundary in Hole 628A. Rare specimens of Discoaster saipanensis occur in samples near the base of the $H$. reticulata Zone in Samples 101$628 \mathrm{~A}-29 \mathrm{X}-2,47-48 \mathrm{~cm}$, and 101-628A-29X-1, 80-81 cm. Their sparseness and poor preservation strongly suggest that these specimens were reworked.

\section{The Sphenolithus predistentus-S. ciperoensis Lineage}

Bramlette and Wilcoxon (1967) were the first to recognize the biostratigraphic utility of sphenoliths in the Oligocene and successfully applied it in naming three mid-Oligocene nannofossil zones. Separation of these zones is based on the evolution of the Sphenolithus predistentus-S. ciperoensis nannofossil lineage. The evolution of this sphenolith lineage provides three important datum levels for nannofossil zonal boundaries in normally low-diversity, mid-Oligocene nannofossil assemblages. These sphenoliths are useful because few other groups of nannofossils undergo significant evolution during this time. Unfortunately, the $S$. predistentus $-S$. ciperoensis lineage undergoes a slow, gradual evolution, and the usefulness of these zones often is masked by the difficulty many workers found when distinguishing the first appearances of cohort species using the light microscope.

Bramlette and Wilcoxon (1967) realized the gradual nature of the evolution of this lineage and noted problems with separation of species in light microscopy, particularly $S$. distentus and $S$. ciperoensis. These sphenolith zones have since been widely used, and other workers noted the gradual evolution of this lineage and associated problems of separation of species using the light microscope (Roth et al., 1971a, 1971b; Haq, 1972; and Lang and Watkins, 1984). Most of these authors specified the difficulty when separating $S$. ciperoensis from $S$. distentus. Haq (1972) recognized this problem, as did Lang and Watkins (1984), who created the informal taxon Sphenolithus sp. cf. S. ciperoensis to include transitional forms. Lang and Watkins also noted a problem with distinguishing Sphenolithus distentus from $S$. predistentus in core material from the Straits of Florida (DSDP Leg 77) because of a number of specimens having an intermediate construction. 
Table 1. Relative abundance, preservation, and distribution of calcareous nannofossils from Hole 628A.

\begin{tabular}{|c|c|c|c|c|c|c|c|c|c|c|c|c|c|c|c|c|c|c|c|c|c|c|c|c|c|c|c|c|c|c|c|}
\hline $\begin{array}{c}\text { Core/section } \\
\text { interval (in cm) }\end{array}$ & 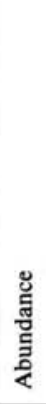 & సัّ & 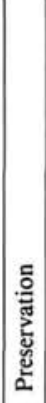 & 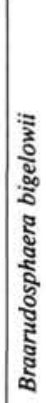 & 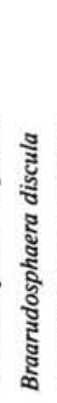 & 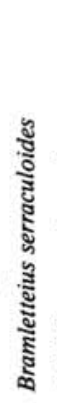 & 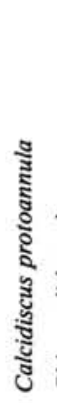 & 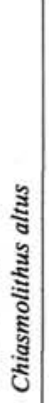 & 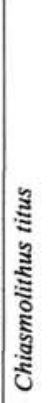 & 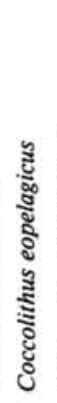 & 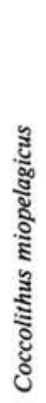 & 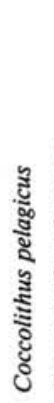 & 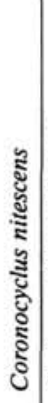 & 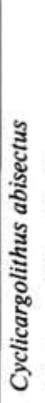 & 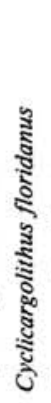 & 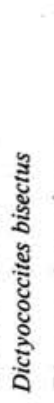 & 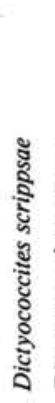 & 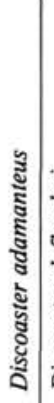 & 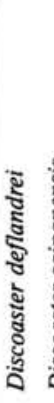 & 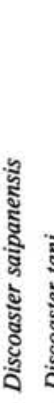 & 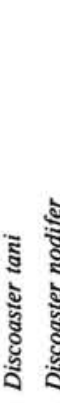 & 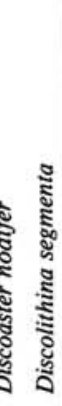 & 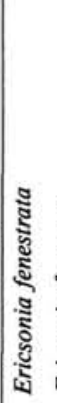 & 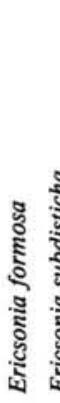 & 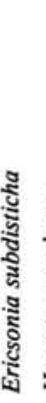 & 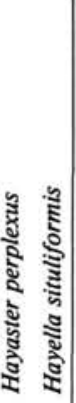 & 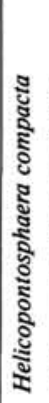 & 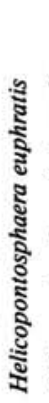 & 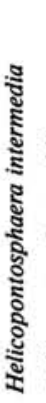 & 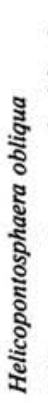 & 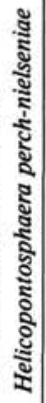 \\
\hline $101-628 \mathrm{~A}-16-1,80-81$ & A & CP19b & G & C & F & & C & & & C & $\mathrm{F}$ & C & C & C & A & $\mathrm{F}$ & $\mathrm{F}$ & & C & & & F & $\mathrm{F}$ & & & F & & C & & $\mathrm{F}$ & \\
\hline $16-3,80-81$ & A & CP19b & $\mathrm{M}$ & $\mathrm{F}$ & $\mathrm{F}$ & & $\mathrm{C}$ & F & & $\mathrm{C}$ & $\mathrm{F}$ & $\mathrm{C}$ & C & C & A & $\mathrm{F}$ & $\mathrm{F}$ & $\mathrm{F}$ & C & & & $\mathrm{F}$ & C & & & C & $?$ & C & $\mathrm{F}$ & C & $\mathrm{F}$ \\
\hline $16-5,80-81$ & $\mathbf{P}$ & CP19b & M & F & $\mathbf{F}$ & & $\mathrm{F}$ & & & C & $\mathrm{F}$ & A & C & C & A & $\mathrm{F}$ & $\mathrm{R}$ & $\mathrm{F}$ & C & & & $\mathrm{F}$ & C & & & C & & C & $\mathbf{F}$ & C & $\mathrm{F}$ \\
\hline $16, \mathrm{CC}$ & $\mathrm{P}$ & CP19b & M & $\mathrm{F}$ & $\mathrm{F}$ & & $\mathrm{F}$ & $\mathrm{F}$ & & $\mathrm{C}$ & F & $\mathrm{C}$ & C & $\mathrm{C}$ & A & $\mathrm{F}$ & & $\mathrm{F}$ & A & & & $\mathrm{F}$ & C & & & & & $\mathrm{C}$ & F & F & $\mathrm{F}$ \\
\hline $17-1,80-81$ & P & $\mathrm{CP} 19 \mathrm{~b}$ & $\mathbf{M}$ & F & $\mathrm{F}$ & & & $\mathbf{R}$ & & C & $\mathrm{F}$ & C & C & C & A & C & $\mathrm{F}$ & $\mathrm{F}$ & C & & & F & C & & & C & & C & $\mathrm{F}$ & C & $\mathbf{F}$ \\
\hline $17-3,80-81$ & A & CP19b & $\mathbf{M}$ & F & F & & $\mathrm{F}$ & & & $\mathrm{F}$ & F & C & $\mathrm{F}$ & C & A & C & F & F & C & & & $\mathrm{F}$ & C & & & C & & C & $\mathrm{F}$ & F & \\
\hline $17-5,80-81$ & A & CP19b & $\mathrm{M}$ & C & $\mathrm{F}$ & & $\mathrm{F}$ & $\mathbf{R}$ & & $\mathrm{C}$ & $\mathrm{F}$ & $\mathrm{C}$ & C & C & A & & $\mathrm{F}$ & $\mathrm{F}$ & C & & & $\mathrm{F}$ & C & & & $\mathrm{F}$ & & C & F & F & \\
\hline $17, \mathrm{CC}$ & A & $\mathrm{CP} 19 \mathrm{~b}$ & $\mathrm{M}$ & C & $\mathrm{F}$ & & $\mathrm{F}$ & & & $\mathrm{C}$ & $\mathrm{F}$ & C & C & C & A & $\mathrm{F}$ & $\mathrm{F}$ & $\mathrm{F}$ & C & & & $\mathrm{F}$ & C & & & F & & C & $\mathrm{F}$ & $\mathrm{F}$ & \\
\hline $18, \mathrm{CC}$ & A & CP19b & $\mathrm{M}$ & C & $\mathrm{F}$ & & $\mathrm{F}$ & & & C & $\mathrm{F}$ & C & C & C & A & $\mathrm{F}$ & $\mathrm{F}$ & & C & & & F & C & & F & $\mathrm{F}$ & & C & F & $\mathrm{F}$ & \\
\hline $19-1,80-81$ & A & CP19b & $\mathrm{M}$ & $\mathrm{F}$ & $\mathrm{F}$ & & $\mathrm{F}$ & $\mathrm{F}$ & & C & $\mathrm{F}$ & C & C & C & A & C & $\mathrm{F}$ & & C & & & F & C & & & $\mathrm{F}$ & & C & $\mathrm{F}$ & F & \\
\hline $19-3,80-81$ & A & $\mathrm{CP} 19 \mathrm{~b}$ & $\mathbf{M}$ & F & F & & $\mathrm{C}$ & F & & C & F & A & C & C & A & C & F & & C & & & F & C & & F & C & & C & C & F & F \\
\hline $20, \mathrm{CC}$ & A & CP19b & M & A & C & & $\mathrm{F}$ & F & & C & & C & C & C & A & C & F & & C & & & F & C & & F & C & & C & F & F & $\mathbf{R}$ \\
\hline $21-1,80-81$ & A & CP19b & $\mathrm{M}$ & C & $\mathrm{F}$ & & C & & & C & $\mathrm{F}$ & A & C & C & A & $\mathrm{F}$ & $\mathrm{F}$ & F & C & & & $\mathrm{F}$ & C & & & & & C & $\mathrm{F}$ & $\mathrm{F}$ & $\mathrm{F}$ \\
\hline $21-3,80-81$ & P & CP19b & M & F & & & C & $\mathbf{R}$ & & C & $F$ & C & C & C & A & C & C & F & C & & & $\mathbf{R}$ & C & & & & & C & $\mathbf{R}$ & F & $\mathrm{F}$ \\
\hline $21, \mathrm{CC}$ & A & CP19b & $\mathrm{M}$ & F & & & $\mathrm{F}$ & & & C & $\mathrm{F}$ & A & C & C & A & $\mathrm{C}$ & C & F & C & & & F & C & & & & & C & $\mathbf{R}$ & F & $\mathbf{R}$ \\
\hline $22-1,80-81$ & A & CP19b & M & $\mathrm{F}$ & F & & C & $\mathrm{R}$ & & $\mathrm{C}$ & $\mathrm{C}$ & C & C & C & A & F & F & $\mathrm{F}$ & C & & $\mathbf{R}$ & F & C & & & $\mathbf{R}$ & & C & F & F & F \\
\hline $22-3,80-81$ & A & CP19b & M & C & $\mathrm{F}$ & & C & $\mathbf{R}$ & & $\mathrm{F}$ & $\mathrm{F}$ & C & C & C & A & C & C & $\mathrm{F}$ & C & & & $\mathrm{F}$ & C & & F & & & C & F & F & $\mathrm{F}$ \\
\hline $22-5,80-81$ & A & CP19b & M & R & & & C & & & $\mathrm{C}$ & $\mathrm{F}$ & C & C & C & A & C & $\mathrm{C}$ & F & C & & & F & C & & F & & & C & $\mathrm{F}$ & F & $\mathbf{R}$ \\
\hline $22, \mathrm{CC}$ & A & CP19b & M & C & F & & $\mathrm{F}$ & F & & C & & C & C & $\mathrm{C}$ & A & C & C & $\mathrm{F}$ & C & & & $\mathrm{F}$ & C & & F & & & C & F & $\mathrm{F}$ & $\mathrm{R}$ \\
\hline $23-1,80-81$ & A & CP19b & M & F & F & & C & & & C & $\mathbf{R}$ & C & C & C & A & C & C & $\mathbf{R}$ & C & & & F & C & & $\mathrm{F}$ & & & C & $\mathrm{F}$ & F & $\mathbf{R}$ \\
\hline $23-2,95-100$ & $?$ & CP19b & P & C & & & & & & C & & C & $\mathrm{F}$ & C & A & $\mathrm{F}$ & C & $\mathrm{F}$ & C & & & & F & & & & & $\mathrm{F}$ & & & \\
\hline $23-3,80-81$ & A & CP19b & M & C & F & & F & & & C & & C & C & C & A & $\mathrm{F}$ & C & $\mathrm{F}$ & C & & & $\mathrm{F}$ & C & & F & & & C & F & & \\
\hline $23-5,80-81$ & A & CP19b & M & C & $\mathrm{F}$ & & $\mathrm{F}$ & F & & C & F & C & C & C & A & $\mathrm{F}$ & & F & C & & & $\mathrm{F}$ & C & & F & F & & C & F & & $\mathrm{F}$ \\
\hline $23, \mathrm{Cl}^{2}$ & A & CP19b & M & C & F & & F & F & & C & F & C & $\mathrm{F}$ & C & A & $\mathrm{F}$ & & $\mathrm{F}$ & C & & & $\mathrm{F}$ & C & & & $\mathbf{R}$ & & C & F & F & \\
\hline $24-1,80-81$ & A & CP19a & G & $\mathrm{F}$ & F & & $\mathrm{C}$ & & & C & $\mathrm{F}$ & C & C & C & A & $\mathrm{F}$ & C & $\mathrm{F}$ & C & & & $\mathrm{F}$ & C & & & & & C & $\mathrm{F}$ & $\mathrm{F}$ & $\mathbf{F}$ \\
\hline $24, \mathrm{CC}$ & A & CP18 & M & C & C & & C & $\mathbf{R}$ & & C & & C & C & C & A & C & C & $\mathrm{F}$ & C & & & F & C & & & & F & C & F & & C \\
\hline $1,80-81$ & A & CP18 & M & C & $\mathrm{F}$ & & C & & & C & & C & C & C & $\mathrm{A}$ & C & C & $\mathrm{F}$ & C & & & $\mathrm{F}$ & C & & F & & F & C & F & & F \\
\hline $25-2,80-81$ & A & CP18 & G & C & $\mathrm{F}$ & & F & F & & C & & C & C & $\mathrm{F}$ & $\mathrm{A}$ & C & $\mathrm{F}$ & $\mathrm{F}$ & C & & & $\mathrm{F}$ & C & & $\mathrm{F}$ & & & C & F & F & $\mathrm{F}$ \\
\hline $25, \mathrm{CC}$ & A & CP17 & G & $\mathrm{F}$ & F & & $\mathrm{F}$ & & & C & & A & $\mathrm{F}$ & $\mathrm{F}$ & $\mathrm{A}$ & C & C & & C & & C & $\mathrm{F}$ & & & C & & C & F & & F & $\mathrm{F}$ \\
\hline $26-1,80-81$ & A & CP17 & $\mathbf{M}$ & C & $\mathrm{F}$ & & $\mathrm{F}$ & & & C & & A & C & & A & C & C & & C & & C & & $\mathbf{R}$ & & & & C & C & $\mathrm{F}$ & & $\mathrm{F}$ \\
\hline $26-2,80-81$ & $P$ & CP17 & G & C & $\mathrm{F}$ & & F & & $?$ & C & & C & $\mathrm{F}$ & $\mathbf{R}$ & A & C & $\mathrm{F}$ & & F & & F & & R & & C & & C & & $\mathbf{R}$ & & \\
\hline & P & CP17 & M & C & $\mathrm{F}$ & $?$ & F & $\mathbf{R}$ & $?$ & C & & C & $\mathrm{F}$ & R & $\mathrm{A}$ & $\mathrm{C}$ & $\mathrm{F}$ & & $\mathrm{F}$ & & $\mathrm{F}$ & & & & $\mathrm{F}$ & & C & & & & \\
\hline $26, \mathrm{CC}$ & P & CP17 & $\mathrm{M}$ & F & F & & & & & $\mathrm{C}$ & & C & $\mathrm{F}$ & $\mathrm{F}$ & A & C & $\mathrm{F}$ & & C & & $\mathrm{F}$ & & $\mathbf{R}$ & & $\mathrm{F}$ & & F & & & F & \\
\hline $27-1,80-81$ & A & CP17 & G & C & $F$ & F & C & $\mathrm{F}$ & & C & & A & C & & A & C & C & & C 1 & $\mathbf{R}$ & C & F & & c & C & $\mathrm{F}$ & A & F & F & & \\
\hline $27-2,80-81$ & A & CP17 & G & C & $\mathrm{F}$ & F & $\mathrm{F}$ & $\mathrm{F}$ & & C & & C & C & & $\mathrm{A}$ & C & $\mathrm{F}$ & & C 1 & R & C & F & & C & C & $\mathbf{R}$ & A & $\mathrm{F}$ & F & & \\
\hline $28-1,80-81$ & A & $\mathrm{CP} 16 \mathrm{~b}$ & M & C & F & C & C & & & C & & A & C & & A & C & $\mathrm{C}$ & & $\mathrm{F}$ & & $\mathbf{F}$ & F & & C C & C & C & C & F & F & & \\
\hline & A & CP16a & M & F & F & C & $\mathrm{C}$ & F & F & C & & A & F & & A & C & C & & C & & C & F & & C & C & C & C & F & F & & \\
\hline $28, \mathrm{CC}$ & P & CP16c & M & C & $\mathrm{F}$ & F & $\mathrm{F}$ & & $\mathrm{F}$ & C & & C & $\mathrm{F}$ & & C & C & $\mathrm{F}$ & & F & $\mathbf{R}$ & F & $\mathrm{F}$ & & $?$ & C & $\mathrm{R}$ & F & $\mathbf{R}$ & & & \\
\hline $29-1,80-81$ & A & CP16a & G & C & $\mathrm{C}$ & C & C & & $\mathrm{C}$ & C & & A & $\mathrm{F}$ & & A & A & C & & C & R & C & $\mathrm{F}$ & & C & C & $\mathrm{C}$ & C & $\mathrm{F}$ & $\mathbf{R}$ & & \\
\hline $29-2,47-48$ & A & CP16a & G & C & $\mathrm{C}$ & C & C & $\mathbf{R}$ & C & C & & C & F & & A & A & C & & C & $\mathbf{R}$ & C & F & & C & C & C & C & F & F & & $\mathbf{R}$ \\
\hline
\end{tabular}

Note: Abundance, $\mathrm{A}=$ abundant, $\mathrm{C}=$ common, $\mathrm{P}=$ periplatform ooze, $\mathrm{F}=$ few, $\mathrm{R}=$ rare, ? = questionable; preservation, $\mathrm{P}=$ poor, $\mathrm{M}=$ moderate, and $\mathrm{G}=$ good.

In addition to the gradual evolution of this group, problems of paleogeography complicate its use. Sphenoliths are more common in lower latitudes than in high latitudes (Edwards, 1971). Perch-Nielsen (1972) suggested that they were more common in relatively shallow-water paleoenvironments, as opposed to deepmarine ("open-ocean") paleoenvironments. In general, these problems suggest the need for more exact taxonomic criteria and/or the use of an alternative set of biostratigraphic indicators.

Identifying the FAD of $S$. distentus was not difficult using material from Hole 628 A because transitional forms between $S$. predistentus and $S$. distentus are rare. As pointed out previously, this may indicate missing material here because of the gradual change other workers noticed between these two species
(Lang and Watkins, 1984). The thinness of the S. distentus Zone and the $C$. floridanus Subzone, as well as the occurrence of several coincident extinctions, also points to an unconformity or unconformities in this part of the section from Hole $628 \mathrm{~A}$.

Separation of $S$. ciperoensis from $S$. distentus in Hole 628A was more difficult, with intermediate forms occurring from Sample 101-628A-24X-1, 80-81 cm, through 101-628A-16H-1, 80$81 \mathrm{~cm}$. Table 2 compares and contrasts various author's descriptions of the structure and morphology of $S$. distentus and $S$. ciperoensis using both light and electron optical systems. A review of the more distinguishing characteristics of both species (as discerned here) is presented in Table 3 . To summarize this table, the length and taper of the apical spine, the width of the proxi- 


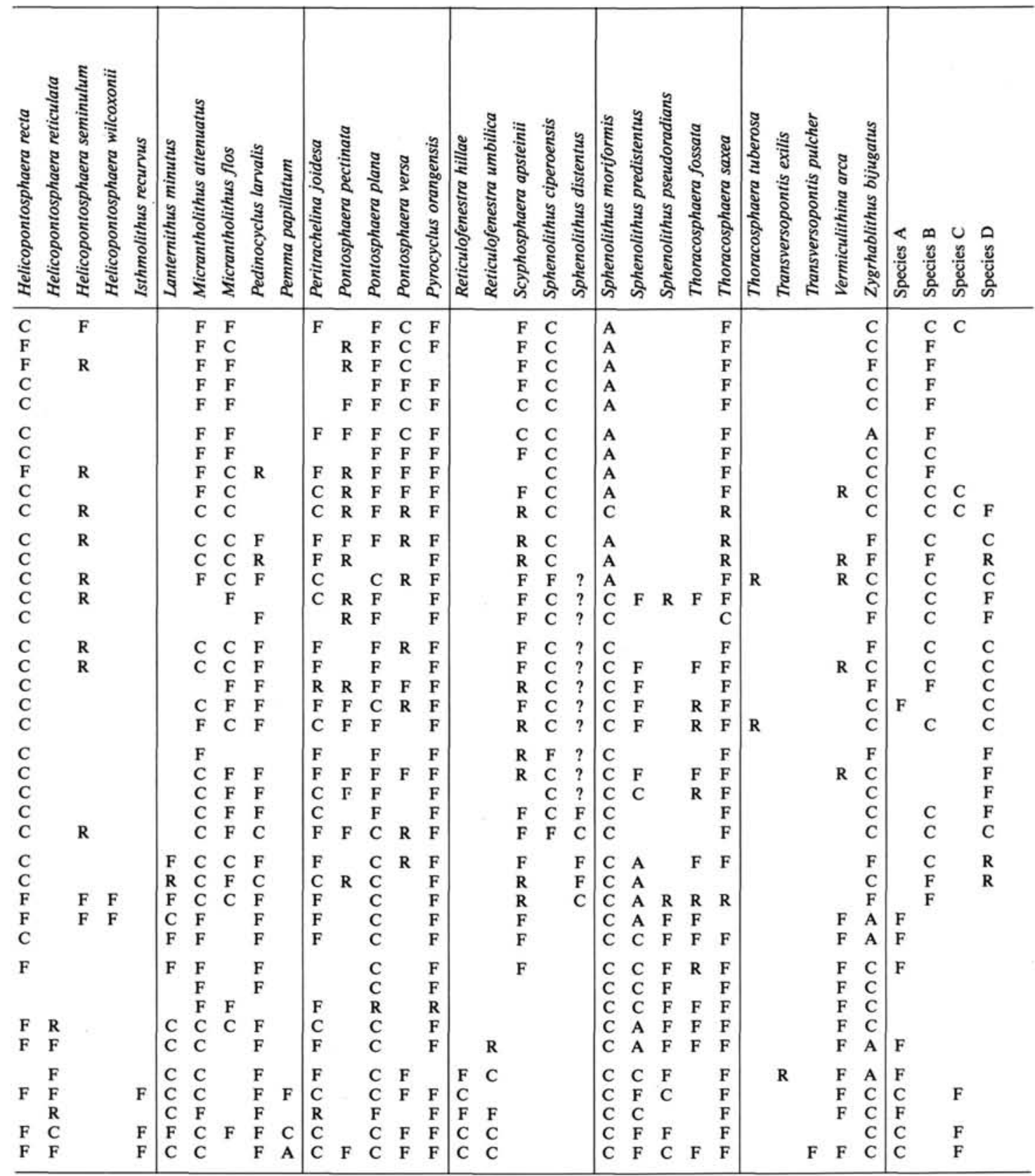

mal shield (basal disc of spines) and the "extinction" line characteristics (interference pattern) of the proximal shield are the most important distinguishing characteristics for separating both species.

Haq (1972) first noticed the characteristic size and taper of the apical spine of $S$. distentus, as opposed to $S$. ciperoensis, using the transmission electron microscope (TEM). The length and taper of the apical spine seem good criteria for separating $S$. ciperoensis from $S$. distentus using light microscopy. However, we found many specimens with an intermediate construction. For example, Sample 101-628-21H-1, 80-81 cm, has a number of specimens with a large apical spine tapering like $S$. distentus, yet the "extinction" lines of the proximal shield (when the apical spine is at $45^{\circ}$ to either polarizer) are characteristic of, or intermediate between, those of $S$. ciperoensis (chevronlike; Bramlette and Wilcoxon, 1967). Throughout the $S$. distentus-S. ciperoensis transition, most of the $S$. ciperoensis exhibit "ex- tinction" lines of the proximal shield intermediate between the two species (Plate 1, Figs. 1 and 4). They also possess variable apical spine lengths, which suggests that the length of the apical spine may not be the most accurate criteria for separating these two species. These intermediate specimens probably represent transitional forms between $S$. distentus and $S$. ciperoensis. They also may represent dimorphic forms of $S$. ciperoensis, with the difference between the two forms being the length and taper of the apical spine.

The most accurate characteristic for separating $S$. ciperoensis from $S$. distentus in material from Hole 628A using light microscopy is the width of the proximal shield. Roth (1970) mentioned the growth of the proximal shield in the evolution from $S$. predistentus to $S$. ciperoensis, with $S$. ciperoensis having the widest proximal shield (basal cycle of elements). This wide proximal shield of $S$. ciperoensis undoubtedly is responsible for its characteristic "extinction" line pattern (chevronlike) in cross- 


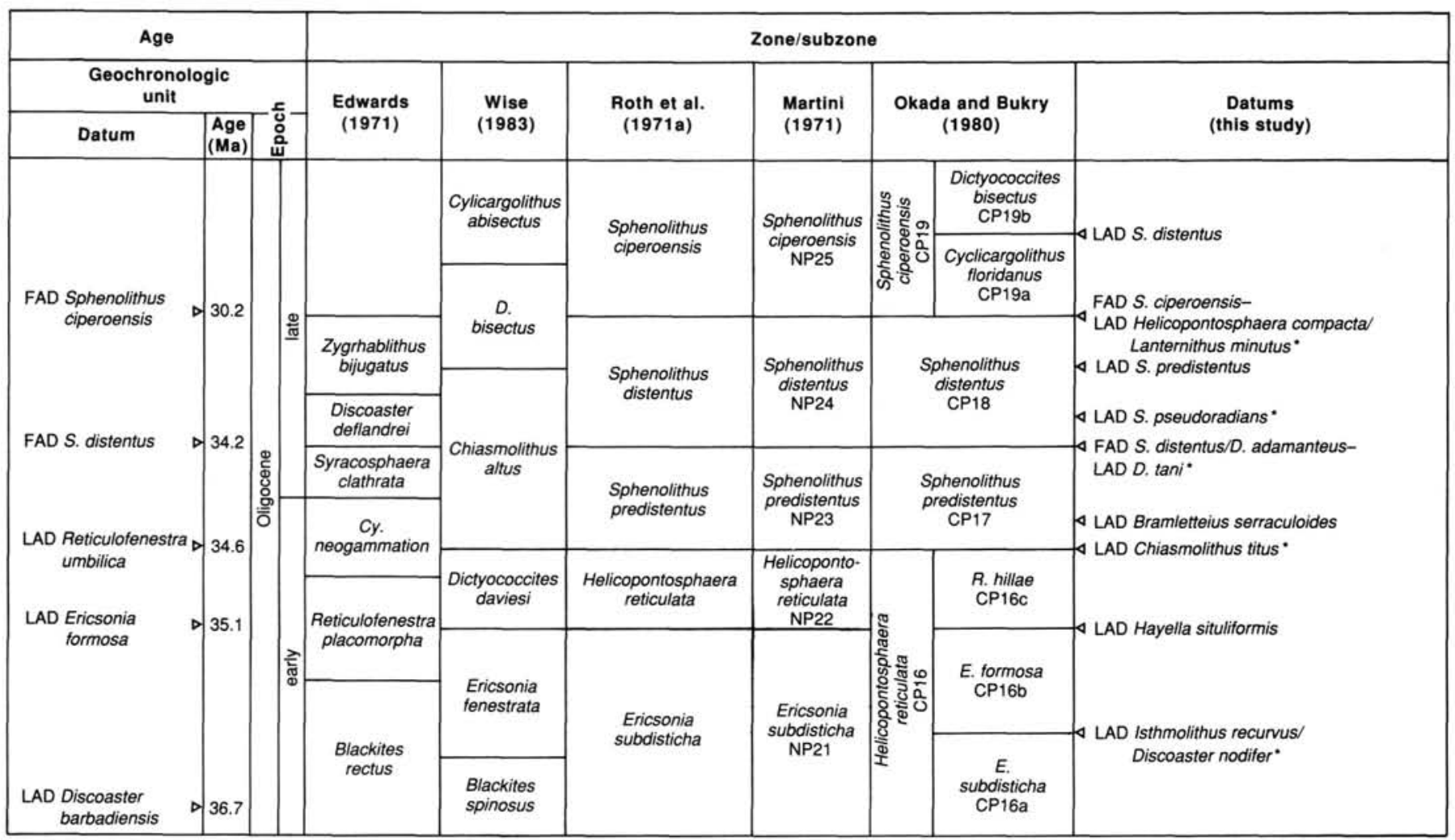

Figure 2. Comparison of Oligocene calcareous-nannofossil zones between high and low latitudes. Roth et al. (1971), Martini (1971), and Okada and Bukry (1980) represent tropical zonations. Edwards (1971) and Wise (1983) are high-latitude zonations. Correlations between tropical zonations and Edwards (1971) are based on the zonation of Martini (1971). Correlation to Wise (1983) is based on the last appearances of Reticulofenestra umbilica and Dictyococcites bisectus. Asterisks indicate low degree of usefulness because of unreliability. Geochronologic age from Berggren et al. (1985). FAD = first-appearance datum; $L A D=$ last-appearance datum.

Table 2. Important diagnostic characteristics of $S$. distentus and $S$. ciperoensis in light and scanning electron microscopy, as described by various authors.

\begin{tabular}{|c|c|c|c|}
\hline & S. distentus & Author & S. ciperoensis \\
\hline \multirow[t]{2}{*}{$\begin{array}{c}\text { Light } \\
\text { microscopy }\end{array}$} & $\begin{array}{l}\text { Apical spine long, } \\
\text { relative to } S \text {. } \\
\text { ciperoensis. }\end{array}$ & $\begin{array}{l}\text { Bramlette and } \\
\text { Wilcoxon } \\
(1967)\end{array}$ & $\begin{array}{l}\text { Apical spine small } \\
\text { with uniform } \\
\text { taper. }\end{array}$ \\
\hline & $\begin{array}{l}\text { "Extinction" lines of } \\
\text { proximal shield } \\
\text { cross when apical } \\
\text { spine is at } 45^{\circ} \text { to } \\
\text { either polarizer. }\end{array}$ & $\begin{array}{l}\text { Bramlette and } \\
\text { Wilcoxon } \\
(1967)\end{array}$ & $\begin{array}{l}\text { "Extinction" lines of } \\
\text { proximal shield } \\
\text { do not cross } \\
\text { (chevronlike) } \\
\text { when apical spine } \\
\text { is at } 45^{\circ} \text { to either } \\
\text { polarizer. }\end{array}$ \\
\hline \multirow[t]{3}{*}{\begin{tabular}{|l} 
Scanning \\
electron \\
microscopy
\end{tabular}} & $\begin{array}{l}\text { Apical spine long from } \\
\text { bifurcated tip to } \\
\text { base. }\end{array}$ & $\begin{array}{l}\text { Roth }(1970) \text { and } \\
\text { Roth et al. } \\
\text { (1971b) }\end{array}$ & $\begin{array}{l}\text { Apical spine short } \\
\text { from bifurcated } \\
\text { tip to base. }\end{array}$ \\
\hline & $\begin{array}{l}\text { Apical spine long; } \\
\text { tapers gradually } \\
\text { near proximal } \\
\text { shield, then } \\
\text { quickly for distal } \\
\text { remainder. }\end{array}$ & Haq (1972) & $\begin{array}{l}\text { Apical spine short } \\
\text { with uniform } \\
\text { taper. }\end{array}$ \\
\hline & $\begin{array}{l}\text { Proximal shield not } \\
\text { much wider than } \\
\text { base of apical } \\
\text { spine. }\end{array}$ & $\begin{array}{l}\text { Roth }(1970) \text { and } \\
\text { Roth et al. } \\
\text { (1971b) }\end{array}$ & $\begin{array}{l}\text { Proximal shield much } \\
\text { wider than base } \\
\text { of apical spine. }\end{array}$ \\
\hline
\end{tabular}

Table 3. Diagnostic morphologic characteristics of $S$. distentus and $S$. ciperoensis (as presented in this study).

\begin{tabular}{|c|c|}
\hline S. distentus & S. ciperoensis \\
\hline $\begin{array}{l}\text { 1. Apical spine generally large; taper- } \\
\text { ing gradual or not at all for } \\
\text { proximal half, than quickly for } \\
\text { distal half (Plate 3, Figs. 1-5). }\end{array}$ & $\begin{array}{l}\text { 1. Apical spine small, with uniform } \\
\text { taper throughout its length } \\
\text { (Plate 1, Figs. 7-9; Plate 2, } \\
\text { Figs. 1-3; Plate 6, Figs. 1-4). }\end{array}$ \\
\hline $\begin{array}{l}\text { 2. Proximal shield rarely wider than } \\
\text { base of apical spine. (Plate 3, Figs. } \\
\text { 1-5). }\end{array}$ & $\begin{array}{l}\text { 2. Proximal shield wider than any } \\
\text { point on apical spine (Plate 1, } \\
\text { Figs. } 8 \text { and 9; Plate 2, Figs. } 2 \\
\text { and 3; Plate 6, Figs. 1-4). }\end{array}$ \\
\hline $\begin{array}{l}\text { 3. "Extinction" lines of proximal } \\
\text { shield are "V"-shaped and cross } \\
\text { when apical spine is at } 45^{\circ} \text { to } \\
\text { either polarizer (Plate 3, Fig. 3). }\end{array}$ & $\begin{array}{l}\text { 3. "Extinction" lines of proximal } \\
\text { shield are "chevronlike" and do } \\
\text { not cross when apical spine is } \\
\text { at } 45^{\circ} \text { to either polarizer (Plate } \\
\text { 1, Fig. 7; Plate 2, Fig. 1). }\end{array}$ \\
\hline
\end{tabular}

polarized light, as described by Bramlette and Wilcoxon (1967). It is this chevronlike "extinction" pattern of the proximal shield that yields the most accurate placement of the first appearance of $S$. ciperoensis. As mentioned previously, many forms that are intermediate between $S$. distentus and $S$. ciperoensis having large apical spines typical of $S$. distentus had proximal shield "extinction" lines diagnostic of $S$. ciperoensis. These transitional forms, as we have called them, caused great confusion regarding the last appearance of $S$. distentus. By strictly using the chevronlike 
"extinction" line pattern (Bramlette and Wilcoxon, 1967) of the proximal shield to categorize $S$. ciperoensis, we were able to place most accurately the last appearance of $S$. distentus in relation to the foraminifer data from Hole 628A (Watkins et al., this volume). Thus, any specimen exhibiting this characteristic proximal shield "extinction" line pattern is termed $S$. ciperoensis regardless of the length of the apical spine.

Others also noticed this increase in width of the proximal shield, most prominently when using the electron microscope. After examination under the TEM, Roth (1970) found that the proximal shield of $S$. ciperoensis was much broader than the base of the apical spine (Table 2). The proximal shield of $S$. distentus was not distinctly wider than the base of the apical spine (Roth, 1970).

In a detailed morphologic study of selected members of the genus Sphenolithus, including $S$. distentus and $S$. ciperoensis, Roth et al. (1971b) concluded, as Roth (1970) did, that the proximal shield (basal disc) of $S$. distentus was equal in width or slightly wider than the base of the apical spine (Plate 2). The proximal shield (basal disc) of $S$. ciperoensis was significantly wider than the base of the apical spine (Roth et al., 1971b). Roth et al. also pointed out the increase in shield/body ratio from $S$. distentus to $S$. ciperoensis. In material from Hole 628A, this increase was also true, with the shield/body ratio for $S$. distentus at around one-fourth, transitional forms between onethird and one-fourth, and the ratio for S. ciperoensis at onethird or less.

Scanning electron micrographs of material from Hole 628A support these findings. $S$. distentus is characterized by a large apical spine composed of two essentially equal-sized elements (Plate 3, Figs. 1-5). The spine is wide proximally, with little or no taper for the proximal half, and then it quickly tapers distally, terminating in a fairly sharp point (Plate 3 , Figs. 1-5). The proximal shield is as wide or only slightly wider than the base of the apical spine where it attaches (Plate 3, Figs. 1 and 2).

Transitional forms between $S$. distentus and $S$. ciperoensis are characterized by having a relatively large apical spine that is similar to that of $S$. distentus (Plate 1, Figs. 1-6; Plate 4, Figs. 3 and 4; Plate 5, Figs. 1-4). The apical spine generally has a characteristic morphology of thinness near its coupling with the proximal shield and then expands rapidly to its widest breadth at approximately one-third its total length. This spine then tapers quickly to a rather sharp point (Plate 4, Figs. 3 and 4; Plate 5 , Figs. 1-4). The proximal shield is generally slightly larger than the base of the apical spine where it attaches and rarely much wider than the widest portion of the apical spine (Plate 4, Figs 3 and 4); Plate 5, Figs. 1-4). Transitional forms can show wide variations in the width of the proximal shield (Plate 4 , Figs. 3 and 4; Plate 5, Figs. 1-4).

Sphenolithus ciperoensis is characterized by having a relatively short, uniformly tapering apical spine consisting of two elements of equal size (Plate 1, Figs. 7-9; Plate 2, Figs. 1-3: Plate 6, Figs. 1-4). The apical spine does not possess the characteristic taper of transitional forms (Plate 6, Figs. 1-4). The proximal shield is distinctly wider than its attachment to the apical spine and any other point on the spine (Plate 6, Figs. 1-4). SEM micrographs of material from Hole $628 \mathrm{~A}$ also clearly show the evolutionary trend in morphology to the wider proximal shield from $S$. predistentus to $S$. ciperoensis (Plate 3, Figs. 1 and 2; Plate 4, Figs. 1 and 2; Plate 6, Figs. 1-4).

When these two species were first described, the existence of forms with a bifurcating apical spine was noted (Bramlette and Wilcoxon, 1967). These were believed to represent dimorphous forms on a single living cell, as in some living coccolithophorids (Bramlette and Wilcoxon, 1967). Towe (1979) speculated that these swallowtail forms surrounded the flagellar pole of his hypothetical "sphenosphere." Although the species Towe (1979) referred to were different, his hypothesis agrees with that of Bramlette and Wilcoxon (1967). Regardless of their biological origins, these forms are not useful here because of their rarity. If these delicately branched specimens do represent dimorphs, as Towe (1979) suggested, then they would be less abundant than nonbifurcated ones, and their biostratigraphic utility would be questionable.

\section{Alternatives to the $S$. ciperoensis FAD}

It is clear that problems still exist when using the first evolutionary appearance of Sphenolithus ciperoensis as a biostratigraphic indicator. Because we have established the slow evolution of the $S$. predistentus-S. ciperoensis lineage by the presence of numerous specimens of intermediate construction between the ideal end-members, these undoubtedly will continue to cause confusion regarding taxonomy and questions about the accuracy of the $S$. ciperoensis FAD. In addition, the sparseness of sphenoliths in higher latitudes (Edwards, 1971) makes their use difficult or impossible in these areas. When seeking possible alternatives to the sphenolith datums, we considered auxiliary nannofossil datums that we (and others) found useful in subdividing and delineating the mid- and upper-Oligocene nannofossil zones.

The LAD of Lanternithus minutus occurs at the first evolutionary appearance (FAD) of $S$. ciperoensis in Section 101-628A24X, CC (Plate 2, Fig. 4; Plate 3, Figs. 6-9). Unfortunately, the LAD of this species may not be useful for approximating the FAD of $S$. ciperoensis for two reasons. First, and most importantly, other workers noted that $L$. minutus has a variable last appearance in time. Roth (1970) indicated that $L$. minutus becomes extinct in the lower part of the Cyclococcolithus margaritae Zone. Roth et al. (1971a) also noted that the upper range of L. minutus extended only into the lower Oligocene at several outcrop areas in Europe. Parker et al. (1985) noted the last appearance of $L$. minutus only into the $S$. predistentus Zone of DSDP Sites 558 and 563 on the west flank of the mid-Atlantic Ridge. Bybell (1982; Fig. 7) reported L. minutus appearing beyond the FAD of $S$. distentus in the lower Oligocene of Alabama and Mississippi. Second, L. minutus often is not found at sites with deeper paleodepths. Okada and Thierstein (1979) did not report the occurrence of $L$. minutus at DSDP Site 386, which contained a fairly thick, complete section of Oligocene. As Site 386 (DSDP Leg 43) was located in $4782 \mathrm{~m}$ of water, it is this water depth that could be the reason that $L$. minutus was not present. As the species is a holococcolith, it is particularly susceptible to dissolution. Great water depths allow a greater chance for dissolution of specimens. In addition, holococcoliths (such as L. minutus) are more susceptible to diagenetic alteration than heterococcoliths. Although Hole 628A was located in only $966 \mathrm{~m}$ of water, still most specimens of $L$. minutus show significant dissolution and/or overgrowth (Plate 3, Figs. 6-9) that make them difficult to recognize using the SEM. As a result of these two factors, the LAD of $L$. minutus, although occurring at the last appearance of $S$. ciperoensis in Hole 628A, is not necessarily useful for approximating this datum.

Only one other datum was observed to correspond to the FAD of S. ciperoensis. Helicopontosphaera compacta becomes extinct (LAD) at the first appearance of $S$. ciperoensis in Section 101-628A-24X, CC (Plate 2, Figs. 5 and 6; Plate 7, Figs. 2 and 4). Bramlette and Wilcoxon (1967) mentioned that this species was rare in the Globigerina opima opima Zone ( $S$. distentus Zone) of the Cipero Section, and they did not show its appearance in the $S$. ciperoensis Zone at all. Roth (1970), however, indicated that this species ranges through his Sphenolithus distentus-S. ciperoensis zone in Alabama, Trinidad, Italy, JOIDES Holes 5 and 6, Mt. Cagnero (Italy), and the Silberg Formation in Helmstedt (northern Germany). Martini (1971) also indicated 
that the LAD of $H$. compacta occurs in his $S$. ciperoensis Zone. Haq (1972) showed that the last appearance of $H$. compacta corresponds to the first appearance of $S$. ciperoensis in the Oligocene of Syria, with only minor overlapping into the overlying zone. This datum helps to define the base of his Sphenolithus distentus-S. ciperoensis Concurrent Range Zone. The last appearance of this species seems somewhat variable, but the large size and relatively easy light-microscope identification could make the LAD of $H$. compacta a good alternative marker for the beginning of the $S$. ciperoensis Zone in some areas. Unfortunately, $H$. compacta is found only at low abundance levels near its LAD in Hole 628A (Table 1) and, thus, may have a low level of recognition.

Another possibility is the use of alternative high-latitude datums for approximating mid-Oligocene Sphenolith datums. Figure 2 illustrates the Oligocene nannofossil zones used by various authors in both high and low latitudes. This figure reflects the changes in floral composition and diversity between tropical and high-latitude nannofossil assemblages during the Oligocene. These changes are a result of greater latitudinal temperature gradients present during this time, which results directly from a cooling trend the whole earth experienced during the Cenozoic.

Hornibrook and Edwards (1971) used a variety of nannoplankton datums to subdivide the middle and upper Oligocene of New Zealand. Some of the datums included are Syracosphaera clathrata (FAD), Chiasmolithus oamaruensis (LAD), Helicopontosphaera obliqua (FAD), and Dictyococcites bisectus (LAD). Edwards (1971) used many of these datums to establish nannofossil zones for the New Zealand Oligocene, where a general absence of many characteristic Oligocene sphenoliths existed (Fig. 2). Martini (1981) used the last appearance of Pontosphaera enormis to approximate the boundary between his NP24 (S. distentus) and NP25 (S. ciperoensis) zones because of sparse sphenoliths. Wise (1983), like Edwards (1971), also used a variety of nannofossil datums (other than sphenoliths) to construct a zonation scheme for the Oligocene in the Falkland Plateau region because of the sparseness of diagnostic nannofossils (Fig. 2).

Most of the high-latitude mid-Oligocene datums proposed by other workers were not useful in the Bahamas. Hornibrook and Edwards (1971) provided several datums below the D. bisectus datum for subdividing the middle Oligocene of New Zealand. From oldest to youngest these include Helicopontosphaera obliqua (FAD), Chiasmolithus oamaruensis (LAD), and Syracosphaera clathrata (FAD). Neither $C$, oamaruensis nor $S$. clathrata were observed in the material studied. Bramlette and Wilcoxon (1967) showed that $H$. obliqua first appears in the Globigerina ciperoensis ciperoensis Zone (S. ciperoensis Zone) of Trinidad. Perch-Nielsen (1985, Fig. 42) also showed this species first appearing in the $S$. ciperoensis Zone. In material from Hole $628 \mathrm{~A}$, H. obliqua first appears is Section 101-628A-26X, CC, which is in the $S$. predistentus Zone. As a result, it is not a good alternative to the $S$. ciperoensis FAD.

Wise (1983) used the last appearances of Ericsonia fenestratus (as Clausicoccus fenestratus), Dictycoccites bisectus, Chiasmolithus altus (last common appearance), and Reticulofenestra umbilica when he subdivided the mid-Oligocene of the Falkland Plateau region. Of these, only the last appearance of $R$. umbilica was observed here (in Section 101-628A-27X, CC). Most of the mid-Oligocene alternative datums developed in higher latitudes simply cannot be applied to lower latitudes. Once again, this reflects the strong nannofossil provincialism occurring at this time because of intense latitudinal temperature gradients. This strong Oligocene provincialism created quite different nannofossil species assemblages between high and low latitudes, which makes it difficult to link useful datums between these areas.

\section{Other Potentially Useful Datum Levels}

A variety of other potentially useful datums were recognized from the Oligocene in Hole 628A and are given in Figure 2. These datums display varying degrees of usefulness, which depends on their consistency in occurrence, as described by various authors.

The first common appearance of Cyclicargolithus abisectus (Sample 101-628A-25X-1, 80-82 cm) closely corresponds to the first appearance of Sphenolithus distentus in Sample 101-628A$25 X-2,80-81 \mathrm{~cm}$. Bramlette and Wilcoxon (1967; Table 1) showed the first appearance of $C$. abisectus (= Coccolithus aff. bisectus; Bramlette and Wilcoxon, 1967) corresponding to the base of the Globigerina opima opima Zone (S. distentus Zone) of the Cipero Section. The FAD of $C$. abisectus defined the base of the $S$. distentus Zone of Roth et al. (1971a). Bukry (1973a) also stated that the first appearance of $C$. abisectus could approximate the first appearance of $S$. distentus at low latitudes. Although the first appearance of $C$. abisectus differs from its first common appearance, the first common appearance may be more reliable as a datum because determining the FAD of this species is quite difficult owing to its slow evolution from Cyclicargolithus floridanus. Like the slow evolution of the $S$. predistentus$S$. ciperioensis lineage, the slow evolution of the mid-Oligocene cyclicargoliths may inhibit their usefulness. Parker et al. (1985) noted a more irregular appearance of $C$. abisectus, with this species occurring commonly in the $H$. reticulata Zone at DSDP Hole 558 and abundantly in this zone in DSDP Hole 563.

The last appearance of Sphenolithus pseudoradians (Sample 101-628A-25X-2, 80-81 cm) and Discoaster tani (Section 101628A-25X, CC) and the first appearance of Discoaster adamanteus (Sample 101-628A-25X-2, 80-81 cm) all correspond closely to the FAD of $S$. distentus. Bramlette and Wilcoxon (1967; Table 2) placed the last appearance of $S$. pseudoradians at the base of the $S$. distentus Zone. However, Parker et al. (1985) showed $S$. pseudoradians extending through the $S$. distentus Zone in DSDP Holes 558 and 563. Perch-Nielsen (1985; Fig. 67) also showed this species range extending into NP24 (CP18). Bramlette and Wilcoxon (1967; Table 2) showed the FAD of $D$. adamanteus at the base of the $S$. distentus Zone. Perch-Nielsen (1985; Fig. 30) placed the first appearance of $D$. adamanteus in CP18 and the last appearance of $D$. tani in the underlying zone CP17. General agreement exists about the close proximity of the above datums with the FAD of $S$. distentus. Where recognized or considered, these datums might be useful for approximating the FAD of $S$. distentus. However, the thinness of the $S$. distentus Zone along with the fact that $S$. pseudoradians is often found into the $S$. distentus Zone raises the possibility that some of the lower part of the $S$. distentus Zone is missing here. This missing material undoubtedly is the result of an unconformity and might cause the great number of species initiation and terminations at this point.

The last appearance of Bramletteius serraculoides occurs within the $S$. predistentus Zone in Sample 101-628A-27X-1, 80-81 cm. Few workers specifically mentioned the last appearance of $B$. serraculoides. This species originally was described from the upper Eocene (Gartner, 1969) and first occurs in the middle Eocene (Gartner, 1971). Gartner (1971) showed this species ranging into the $H$. reticulata Zone of the lower Oligocene but did not indicate its last appearance. Bybell (1982) showed B. serraculoides becoming extinct in the Red Bluff and Bumpnose formations (early Oligocene) in Alabama. Parker et al. (1985) reported this species as last appearing in the $R$. hillae Subzone of DSDP Hole 558 and in the lower part of the $S$. predistentus Zone of DSDP Hole 563. Perch-Nielsen (1985; Fig. 19) showed this species ranging through CP16.

The last appearance of Chiasmolithus titus occurs in Sample 101-628A-27X-2, 80-81 cm. Chiasmolithus titus was first de- 
scribed by Gartner (1970) as occurring from the middle Eocene through the upper Eocene. He noted that the species is well developed in the Shubuta Clay Member of the Yazoo Clay (upper Eocene) in Mississippi. Bybell (1982) also showed this species ranging into the Shubuta Member, but no higher. Perch-Nielsen (1985; Fig.19) showed the range of this species extending into CP16A. C. titus only occurs sporadically and in low abundance levels through the lower Oligocene of Hole 628A. Gartner (1970) mentioned the sporadic occurrence of this species throughout its range. Therefore, its LAD may be unrecognizable and, thus, not very useful.

The LAD of the distinctive Hayella situliformis (Plate 7, Fig. 3) closely coincides with that of Ericsonia formosa in Section 101-628A-27X, CC. Where present, this extinction may help mark an upper boundary for the $E$. formosa Subzone (CP16b). Gartner (1971) documented the last appearance of this species in the $H$. reticulata Zone of JOIDES Cores J-3 and J-6B but did not mention it as a datum. Bybell (1982; Fig. 7) also reported the last appearance of $H$. situliformis in the lower Oligocene of Alabama and Mississippi but did not mention this as a biohorizon. Hayella situliformis is never abundant in the $H$. reticulata Zone of Hole 628A but is easily recognizable under the light microscope because of the high birefringence of its upper and lower rims, making it a potentially useful biostratigraphic indicator.

Both the LADs of Isthmolithus recurvus and Discoaster nodifer occur at the base of the E. formosa Subzone (CP16b; Sample 101-628A-28X-2, $80-81 \mathrm{~cm}$ ). These datums may be useful for subdividing the lower part of the $H$. reticulata Zone, where the acme of $E$. subdisticha was not observed. Roth et al. (1970) reported that the LAD of $I$. recurvus occurred within their Cyclococclithus margaritae Zone. They also noted that this species became extinct near the top of their $E$. subdisticha Zone (CP16a; Okada and Bukry, 1980) or in the lower part of their $H$. reticulata Zone (CP16; Okada and Bukry, 1980). Martini (1971; Table 1) indicated that $I$. recurvus has its last appearance just below the top of NP22 (= top of CP16c). Bukry (1973a) reported that $I$. recurvus is extinct in the Ericsonia formosa Subzone (CP16b) and is present in the underlying Ericsonia subdisticha Zone (CP16a). Perch-Nielsen (1985; Fig. 78) showed this species becoming extinct in CP16c. Thus, general agreement exists that the LAD of I. recurvus is at or near the boundary of CP16b and CP16c.

Bramlette and Wilcoxon (1967) reported the last appearance of Discoaster nodifer at the base of the Globigerina opima opima Zone ( $S$. distentus Zone). Roth et al., (1971a) showed this species ranging into their $H$. reticulata zone and, once, into the lower part of the $S$. predistentus Zone. Martini (1971; Table 1) indicated that $D$. nodifer (as $D$. tani nodifer) extends only into his E. subdisticha Zone. Bukry (1973b) indicated that Discoaster nodifer occurs from the upper Eocene through the $S$. ciperoensis Zone in the eastern equatorial Pacific. Bybell (1982; Fig. 7) also showed the range of this species extending beyond the FAD of $S$. distentus in Alabama and Mississippi. The LAD of $D$. nodifer is quite variable, and its usefulness as a biohorizon is questionable.

\section{SUMMARY AND CONCLUSIONS}

The calcareous nannofossils from Hole 628A yielded valuable information regarding Oligocene nannofossil biostratigraphy. The thickness of the $S$. ciperoensis Zone allowed us to examine the biostratigraphically important $S$. distentus-S. ciperoensis transition in detail. Initially, we thought that the length and taper of the apical spine were the most distinguishing characteristics between these two species. However, transitional forms between $S$. distentus and $S$. ciperoensis that have large apical spines like $S$. distentus also tend to have variable proximal shield widths. Often these shields are wide enough to produce "extinction" patterns diagnostic of $S$. ciperoensis when viewed in crosspolarized light. This caused confusion regarding the last appearance of $S$. distentus. Using light microscopy we found that the "extinction" line pattern of the proximal shield was the best characteristic for placing the FAD of $S$. ciperoensis and the LAD of $S$. distentus because it correlated most accurately with other microfossil dating. $S$. distentus has proximal shield "extinction" lines that are "V"-shaped and cross when the apical spine is at $45^{\circ}$ to either polarizer (Plate 3, Fig. 3; Table 3), while in $S$. ciperoensis the "extinction" lines are chevronlike (Bramlette and Wilcoxon, 1967) and do not cross (Plate 1, Fig. 7; Plate 2, Fig. 1; Table 3). Any specimen that exhibits the $S$. ciperoensis proximal shield "extinction" lines should be considered as such regardless of the length of the apical spine.

SEM confirmed that the proximal shield "extinction" line pattern results from growth of the proximal shield during evolution from $S$. distentus to $S$. ciperoensis. Sphenolithus distentus is characterized by having a proximal shield that is only as wide, or slightly wider, than the base of the apical spine where it attaches (Plate 3, Fig. 1 and 2). S. ciperoensis has a proximal shield that is distinctly wider than the base of the apical spine or any other point on the spine (Plate 6, Figs 1-4). Transitional forms have relatively large apical spines, like $S$. distentus, but their proximal shields exhibit large variations in width, many of which are as wide as in S. ciperoensis (Plate 4, Figs. 3 and 4; Plate 5, Figs. 1-5).

The last appearances of other species, such as Lanternithus minutus and Helicopontosphaera compacta, correspond with the FAD of $S$. ciperoensis in Hole $628 \mathrm{~A}$, and we considered them as possible alternatives to it. The LAD of $H$. compacta may be the most useful datum for approximating the FAD of $S$. ciperoensis; however, some workers have noticed a somewhat variable LAD of this species, which may have a low level of recognition near its last appearance because of low abundance levels. The LAD of $L$. minutus is not a useful datum because of its susceptibility to dissolution at depth, its susceptibility to diagenetic alteration, and the variability of its last appearance.

Finally, transitional forms may represent an intermediate evolutionary form between $S$. distentus and $S$. ciperoensis as a result of sluggish evolution. A continuum of forms of intermediate construction between ideal end-members are to be expected in a lineage that undergoes such a gradual evolution. These transitional forms also may represent dimorphic forms on the same cell, or they simply may be the result of syntaxial overgrowth of short-spined S. ciperoensis. Even less likely, they may represent a different (biological) species. In any case, placing these intermediate forms in a well-defined category will always be difficult. However, if the criteria set forth here are adhered to, results should be better.

\section{ACKNOWLEDGMENTS}

We thank the Ocean Drilling Program and the U.S. Scientific Advisory Committee (USSAC) for financial support in completing this project. Support from the Department of Geology, University of Nebraska, also is gratefully acknowledged. We thank James A. Bergen and John C. Steinmetz for reviewing the manuscript and for offering suggestions that significantly improved the final draft. J. A. Austin, Jr., A. A. Palmer, and W. Schlager also reviewed the manuscript. Michael J. Moran gives special thanks to Linda Watts for helping him get through both the good and the bad while he produced this study.

\section{REFERENCES}

Austin, J. A., Jr., Schlager, W., et al., 1986. Proc. ODP, Init. Repts., 101: College Station, TX (Ocean Drilling Program).

Berggren, W. A., Kent, D. V., and Flynn, J. J., 1985. Jurassic to Paleogene: part 2, Paleogene geochronology and chronostratigraphy. In Snelling, N. J. (Ed.), The Chronology of the Geologic Record: (Blackwell Scientific Publications), 141-195. 
Bramlette, M. N., and Wilcoxon, J. A., 1967. Middle Tertiary calcareous nannoplankton of the Cipero Section, Trinidad, W. I. Tulane Stud. Geol. Paleontol., 5:93-131.

Bukry, D., 1973a. Low-latitude coccolith biostratigraphic zonation. In Edgar, N. T., Saunders, J. B., et al., Init. Repts. DSDP, 15: Washington (U.S. Govt. Printing Office), 685-703. 1973b. Coccolith stratigraphy, Leg 10-Deep Sea Drilling Project. In Worzel, J. L., Bryant, W., et al., Init. Repts. DSDP, 10: Washington (U.S. Govt Printing Office), 385-406.

1975. Coccolith and silicoflagellate stratigraphy, northwestern Pacific Ocean, Deep Sea Drilling Project Leg 32. In Larson, R. L., Moberly, R., et al., Init. Repts. DSDP, 32: Washington (U.S. Govt. Printing Office), 677-701.

Bybell, L. M., 1982. Late Eocene to early Oligocene calcareous nannofossils in Alabama and Mississippi. Gulf Coast Assoc. Geol. Soc. Trans., 32:295-302.

Edwards, A. R., 1971. A calcareous nannoplankton zonation of the New Zealand Paleogene. In Farinacci, A. (Ed.), Proc. 2nd Plankt. Conf. Roma: Rome (Edizioni Tecnoscienza), 2:381-419.

Gartner, S., 1969. Two new calcareous nannofossils from the Gulf Coast Eocene. Micropaleontology, 15:31-34.

Gartner, S., 1970. Phylogenetic lineage in the lower Tertiary coccolith genus Chiasmolithus. Proc. North American Paleontol. Conv., 930-957.

1971. Calcareous nannofossils from the JOIDES Blake Plateau cores and revision of Paleogene nannofossil zonation. Tulane Stud. Geol. Paleontol., 8:101-121.

Haq, B. U., 1972. Paleogene calcareous nannoflora, Part III: Oligocene of Syria. Stockholm Contrib. Geol., 21:99-127.

Hay, W. W., 1970. Calcareous nannofossils from cores recovered on Leg 4. In Bader, R. G., Gerard, R. D., et al., Init. Repts. DSDP, 4: Washington (U.S. Govt Printing Office), 455-501.

Hornibrook, N. B., and Edwards, A. R., 1971. Integrated planktonic foraminiferal and calcareous nannoplankton datum levels in the New Zealand Cenozoic. In Farinacci, A. (Ed.), Proc. 2nd Plankt. Conf. Roma: Rome (Edizioni Tecnoscienza), 2:649-657.

Lang, T. H., and Watkins, D. K., 1984. Cenozoic calcareous nannofossils from Deep Sea Drilling Project Leg 77: biostratigraphy and delineation of hiatuses. In Buffler, R. T., Schlager, W., et al., Init. Repts. DSDP, 77: Washington (U.S. Govt. Printing Office), 629648.

Martini, E., 1971. Standard Tertiary and Quaternary calcareous nannoplankton zonation. In Farinacci, A. (Ed.), Proc. 2nd Plankt. Conf. Roma: Rome (Edizioni Tecnoscienza), 2:739-785.

Martini, E., 1981. Nannoplankton in der Ober-Kriede, im Alttertiär und im tieferen Jungtertiär von Süddeutchland um dem angrenzenden österreich. Geol. Bavar., 82:345-356.

Okada, H., and Thierstein, H. R., 1979. Calcareous nannoplanktonLeg 43, Deep Sea Drilling Project. In Tucholke, B. E., Vogt, P. R., et al., 1979. Init. Repts. DSDP, 43: Washington (U.S. Govt. Printing Office), 507-573.

Okada, H., and Bukry, D., 1980. Supplementary modification and introduction of code numbers to the "low-latitude coccolith biostratigraphic zonation” (Bukry 1973; 1975). Mar. Micropaleontol., 5: 321-325.

Parker, M. E., Clark, M., and Wise, S. W., 1985. Calcareous nannofossils of Deep Sea Drilling Project Sites 558 and 563, North Atlantic Ocean: biostratigraphy and the distribution of Oligocene braarudosphaerids. In Bougault, H., Cande, S. C., et al., Init. Repts. DSDP, 92: Washington (U.S. Govt. Printing Office), 559-589.

Perch-Nielsen, K., 1972. Remarks on Late Cretaceous to Pleistocene coccoliths from the North Atlantic. In Laughton, A. S., Berggren, W. A., et al., Init. Repts. DSDP, 12: Washington (U.S. Govt. Printing Office), 1003-1069.

1985. Cenozoic calcareous nannofossils. In Bolli, H. M., Saunders, J. B., and Perch-Nielsen, K. (Eds.), Plankton Stratigraphy: Cambridge (Cambridge Univ. Press), 427-554.

Roth, P. H., 1970. Oligocene calcareous nannoplankton biostratigraphy. Ecologae Geol. Helvetiae, 63:799-881.

Roth, P. H., and Hay, W. W., 1967. Zonation of the Oligocene interval. In Hay, W. W., Mohler, H. P., Roth, P. H., Schmidt, R. R., and Boudreaux, J. E. Calcareous nannoplankton zonation of the Cenozoic of the Gulf Coast and Caribbean-Antillean area, and transoceanic correlation. Gulf Assoc. Geol. Socs. Trans., 17:439-480.
Roth, P. H., Baumann, P., and Bertolino, V., 1971a. Late Eocene-Oligocene calcareous nannoplankton from central and northern Italy. In Farinacci, A. (Ed.), Proc. 2nd Plankt. Conf. Roma; Rome (Edizioni Tecnoscienza), 2:1069-1097.

Roth, P. H., Franz, H. E., and Wise, S. W., 1971b. Morphological study of selected members of the genus Sphenolithus Deflandre (Incertae Sedis, Tertiary). In Farinacci, A. (Ed.), Proc. 2nd Plankt. Conf. Roma: Rome (Edizioni Tecnoscienza), 2:1099-1019.

Towe, K. M., 1979. Variation and systematics in calcareous nannofossils of the genus Sphenolithus. Am. Zool., 19:555-572.

Watkins, D. K., and Bowdler, J. L., 1984. Cretaceous calcareous nannofossils from Deep Sea Drilling Project Leg 77, southeast Gulf of Mexico. In Buffler, R. T., Schlager, W., et al., Init. Repts. DSDP, 77: Washington (U.S. Govt. Printing Office), 649-674.

Wise, S. W., Jr., 1983. Mesozoic and Cenzoic calcareous nannofossils recovered by Deep Sea drilling Project Leg 71 in the Falkland Plateau region, southwest Atlantic Ocean. In Ludwig, W. J., Krasheninnikov, V. A., et al., Init. Repts, DSDP, 71: Washington (U.S. Govt. Printing Office), 481-550.

Date of initial receipt: 25 November 1986

Date of acceptance: 11 June 1987

Ms 101B-174

\section{APPENDIX}

Calcareous Nannofossil Taxa Considered (listed alphabetically by species epithet)

Cyclicargolithus abisectus (Müller, 1970) Wise 1973

Discoaster adamanteus Bramlette and Wilcoxon (1967)

Chiasmolithus altus Bukry and Percival (1971)

Scyphosphaera apsteinii Lohmann (1902)

Micrantholithus attenuatus Bramlette and Sullivan (1961)

Vermiculithina arca Bukry and Percival (1971)

Braarudosphaera bigelowii (Gran and Braarud, 1935) Deflandre (1947)

Zygrhablithus bijugatus (Deflandre in Deflandre and Fert, 1954) Deflandre (1959)

Dictyococcites bisectus (Hay, Mohler, and Wade, 1966) Bukry and Percival (1971)

Sphenolithus ciperoensis Bramlette and Wilcoxon (1967)

Helicopontosphaera compacta Bramlette and Wilcoxon (1967)

Discoaster deflandrei Bramlette and Riedel (1954)

Braarudosphaera discula Bramlette and Riedel (1954)

Sphenolithus distentus (Martini, 1965) Bramlette and Wilcoxon (1967)

Coccolithus eopelaqicus (Bramlette and Riedel, 1954) Bramlette and Sullivan (1961)

Helicopontosphaera euphratis Haq (1966)

Transversopontis exilis (Bramlette and Sullivan, 1961) Perch-Nielsen (1971)

Ericsonia fenestrata (Deflandre and Fert, 1954) Stradner in Stradner and Edwards (1968)

Cyclicargolithus floridanus (Roth and Hay in Hay et al., 1967) Bukry (1971)

Micrantholithus flos Deflandre in Deflandre and Fert (1954)

Ericsonia formosa (Kamptner, 1963) Haq (1971)

Thoracosphaera fossata Jafar (1975)

Reticulofenestra hillae Bukry and Percival (1971)

Helicopontosphaera intermedia Martini (1965)

Peritrachelina joidesa Bukry and Bramlette (1968)

Pedinocyclus larvalis (Bukry and Bramlette, 1969) Loeblich and Tappan (1973)

Lanternithus minutus Stradner (1962)

Coccolithus miocelagicus Bukry (1971)

Sphenolithus moriformis (Brönnimann and Stradner, 1960) Bramlette and Wilcoxon (1967)

Coronocyclus nitescens (Kamptner, 1963) Bramlette and Wilcoxon (1967)

Discoaster nodifer (Bramlette and Riedel, 1954) Bukry (1973)

Helicopontosphaera obliqua Bramlette and Wilcoxon (1967)

Pyroyclus orangensis (Bukry, 1971) Backman (1980)

Pemma papillatum Martini (1959)

Pontosphaera pectinata (Bramlette and Sullivan, 1961) Sherwood (1974)

Coccolithus pelagicus (Wallich, 1877) Schiller (1930)

Helicopontosphaera perch-nielseniae Haq (1971)

Hayaster perplexus (Bramlette and Riedel, 1954) Bukry (1973) 
Pontosphaera plana (Bramlette and Sullivan, 1961) Haq (1971) Sphenolithus predistentus Bramlette and Wilcoxon (1967)

Calcidiscus protoannula (Gartner, 1971) Loeblich and Tappan (1978)

Sphenolithus pseudoradians Bramlette and Wilcoxon (1967)

Transversopontis pulcher (Deflandre in Deflandre and Fert, 1954) Perch-

\section{Nielsen (1967)}

\section{Helicopontosphaera recta $\mathrm{Haq}(1966)$}

Isthmolithus recurvus Deflandre (1954)

Helicopontosphaera reticulata Bramlette and Wilcoxon (1967)

Discoaster saipanensis Bramlette and Riedel (1954)

Thoracosphaera saxea Stradner (1961)

Dictyococcites scripssae Bukry and Percival, 1971
Discolithina segmenta Bukry and Percival (1971)

Helicopontosphaera seminulum Bramlette and Sullivan (1961)

Bramletteius serraculoides Gartner (1969)

Hayella situliformis Gartner (1969)

Ericsonia subdisticha (Roth and Hay in Hay et al., 1967) Roth in Baumann and Roth (1969)

Discoaster tani Bramlette and Riedel (1954)

Chiasmolithus titus Gartner (1970)

Thoracosphaera tuberosa Kamptner (1963)

Reticulofenestra umbilica (Levin, 1965) Martini and Ritzkowski (1968)

Pontosphaera versa (Bramlette and Sullivan, 1961) Sherwood (1974)

Helicopontosphaera wilcoxonii Gartner (1971)

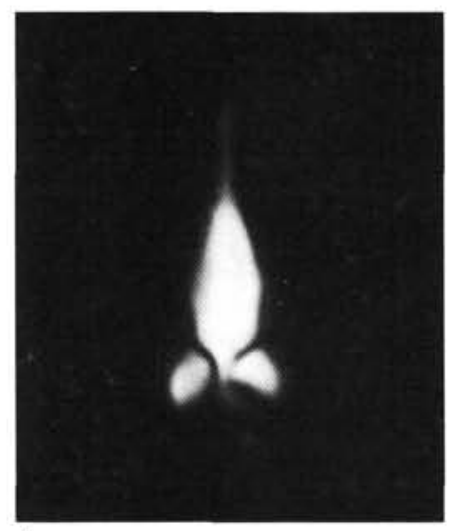

1

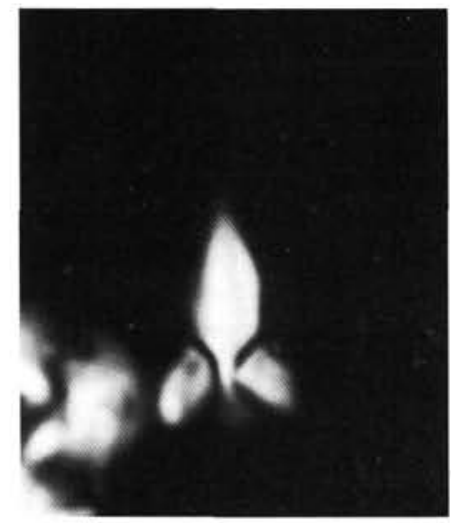

4

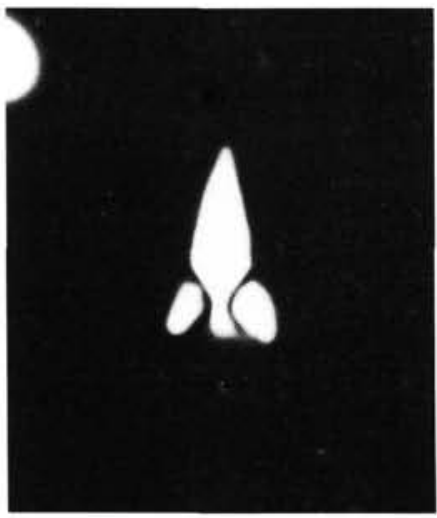

7

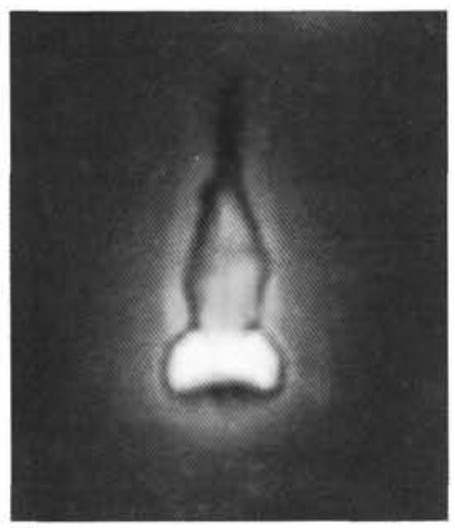

2

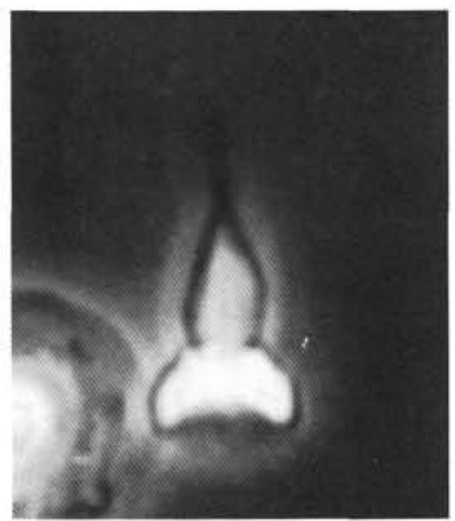

5

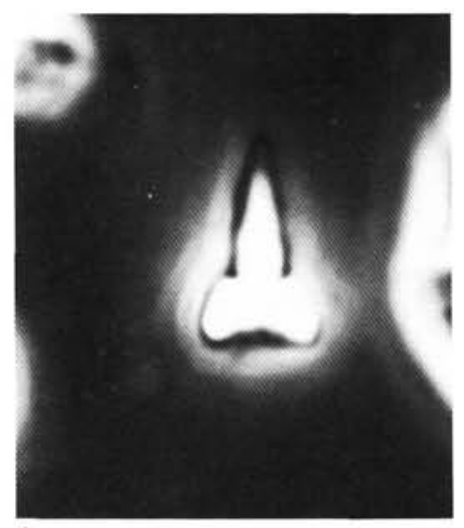

8

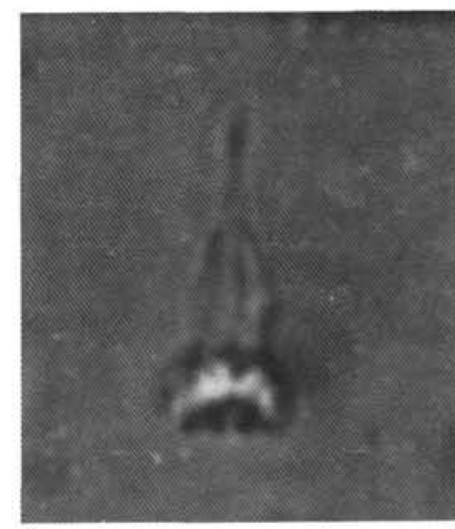

3

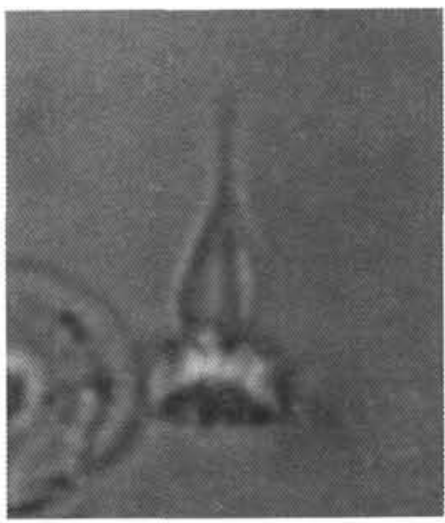

6

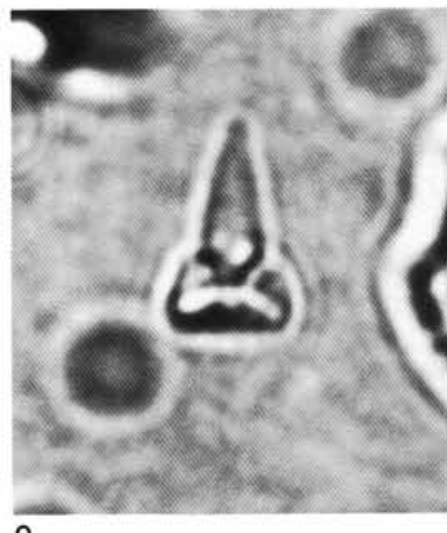

9

Plate 1. All specimens $\times 4125$ and from Sample 101-628A-21H-1, 80-81 cm. 1-3. Transitional form between Sphenolithus distentus and Sphenolithus ciperoensis; (1) cross-polarized light, apical spine at $45^{\circ}$ to either polarizer; (2) phase contrast; (3) transmitted light. 4-6. Transitional form; (4) cross-polarized light, apical spine at $45^{\circ}$ to either polarizer; (5) phase contrast; (6) transmitted light. 7-9. Sphenolithus ciperoensis; (7) cross-polarized light, apical spine at $45^{\circ}$ to either polarizer; (8) phase contrast; (9) transmitted light. 


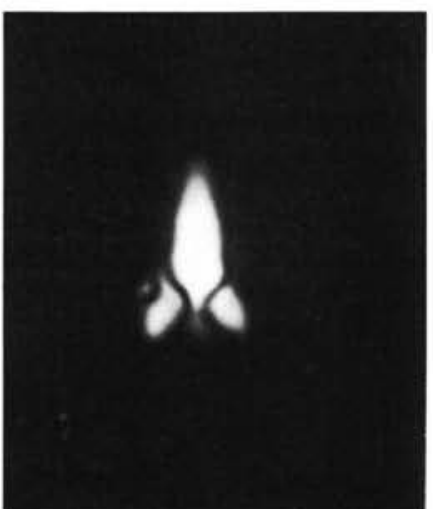

1

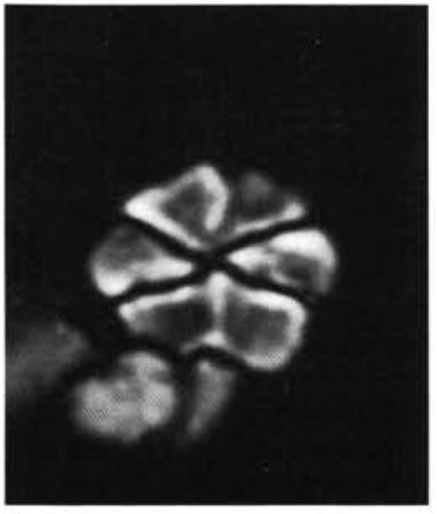

4

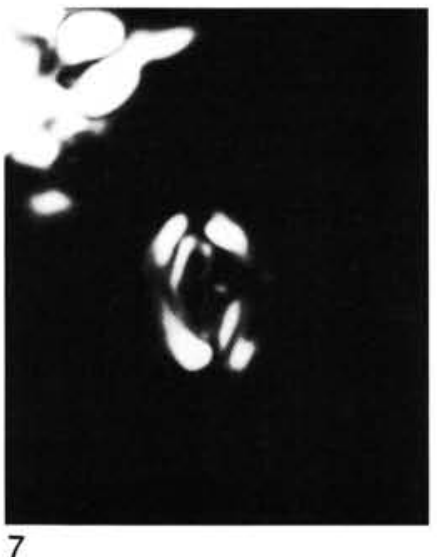

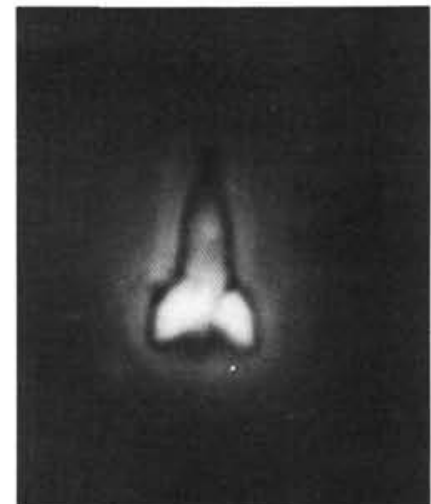

2

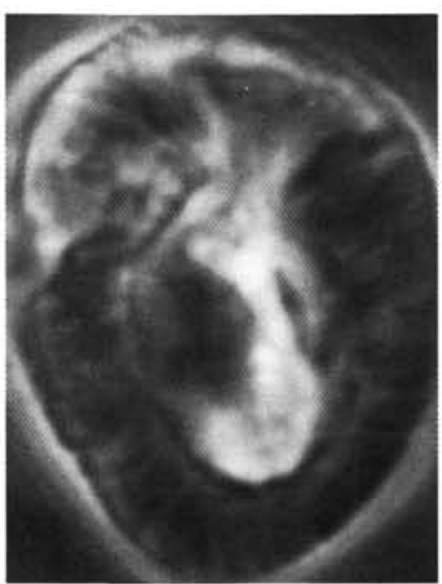

5

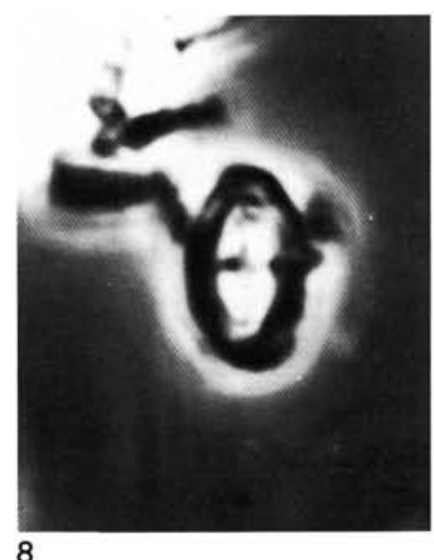

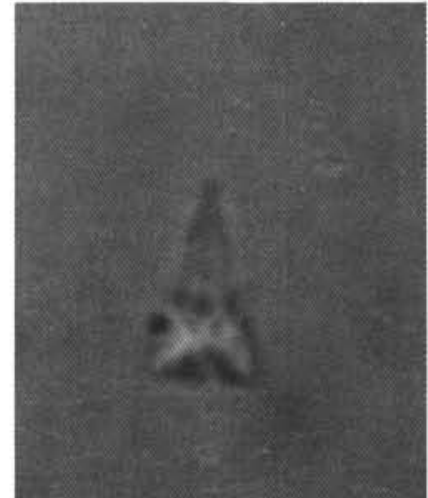

3

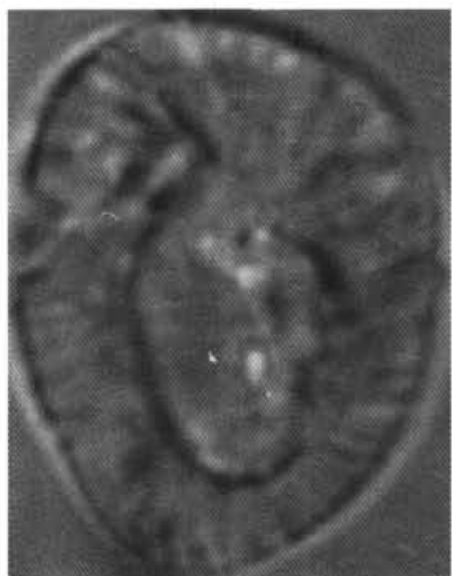

6

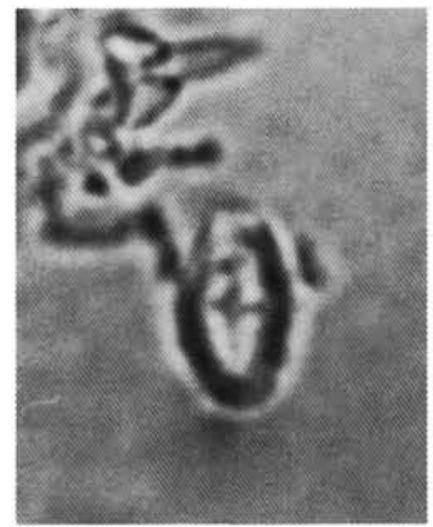

9

Plate 2. All specimens $\times 4125$. 1-3. Sphenolithus ciperoensis, Sample 101-628A-21H-1, 80-81 cm; (1) cross-polarized light, apical spine at $45^{\circ}$ to either polarizer; (2) phase contrast; (3) transmitted light. 4. Lanternithus minutus, cross-polarized light, Section 101-628A-25X, CC. 5-6. Helicopontosphaera compacta, Section 101-628A-25X, CC; (5) phase contrast; (6) transmitted light. 7-9. Species D, Sample 101-628A-24X-1, 80-81 cm; (7) cross-polarized light; (8) phase contrast; (9) transmitted light. 

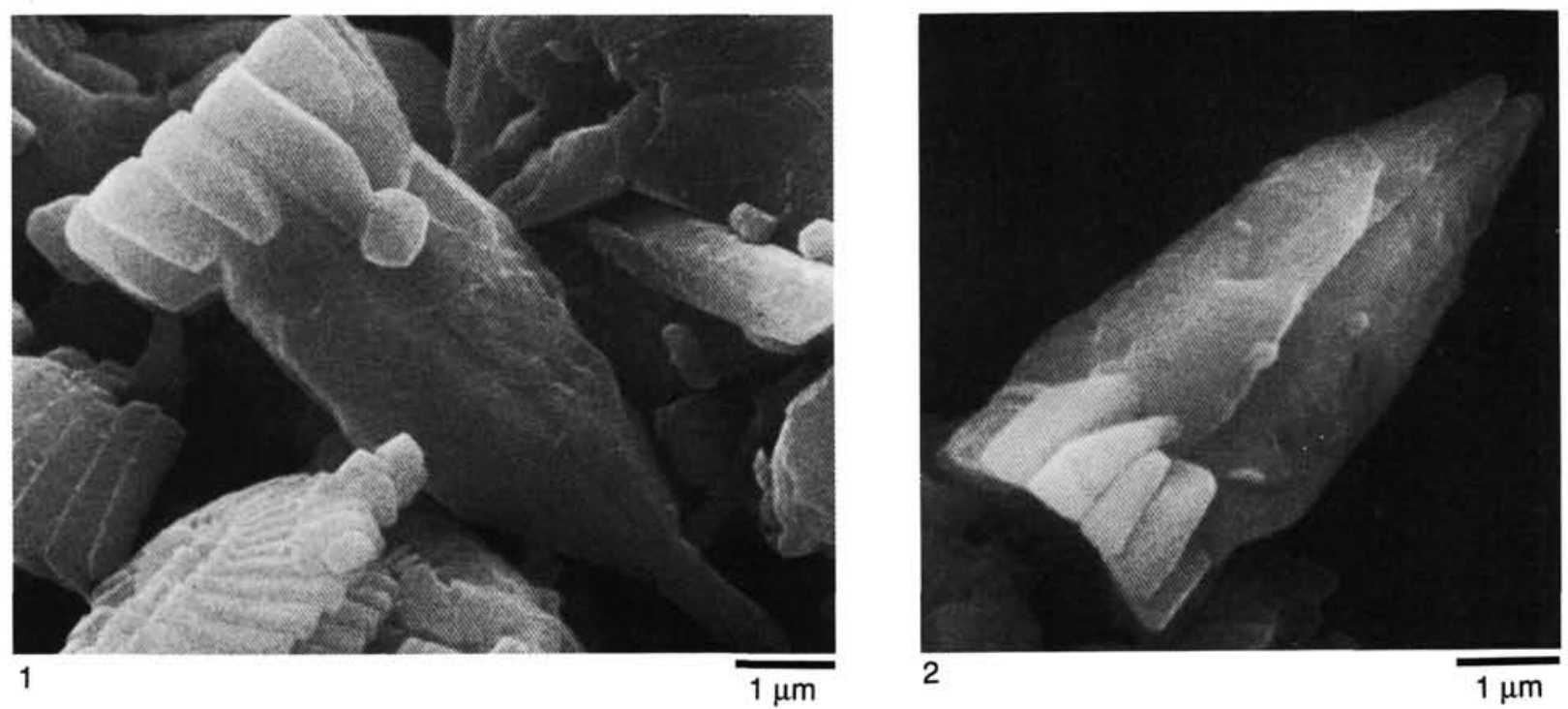

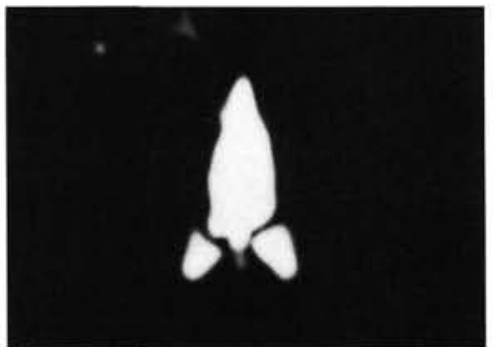

3

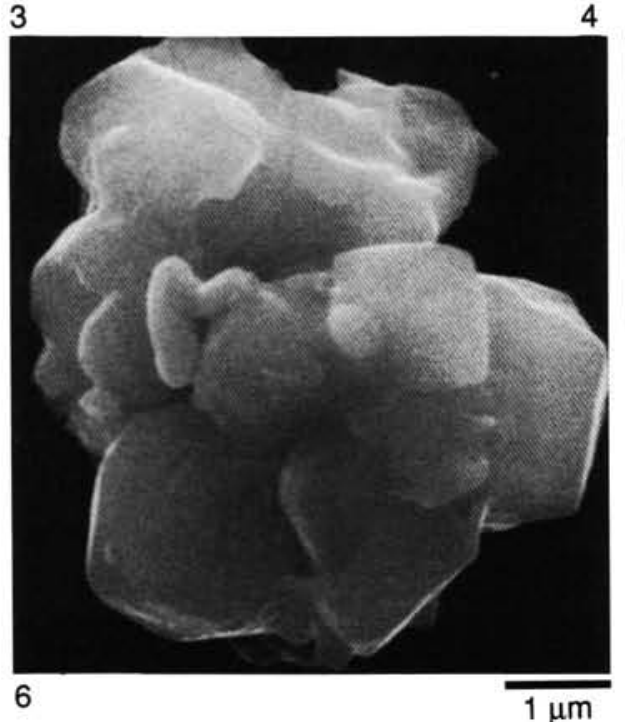

$1 \mu \mathrm{m}$

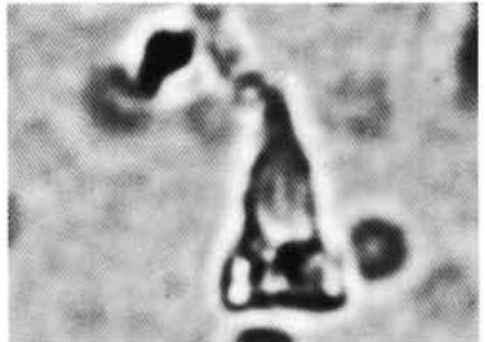

4

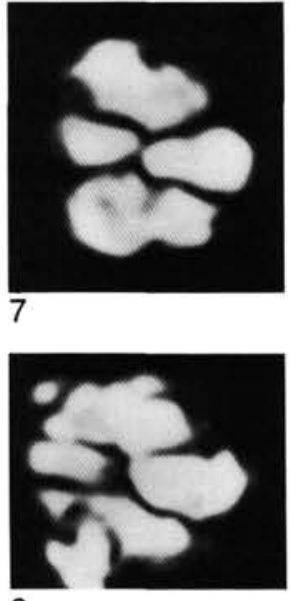

9

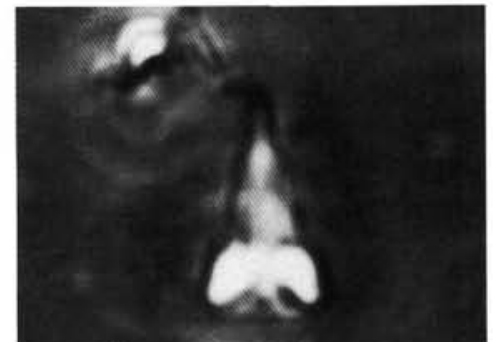

5

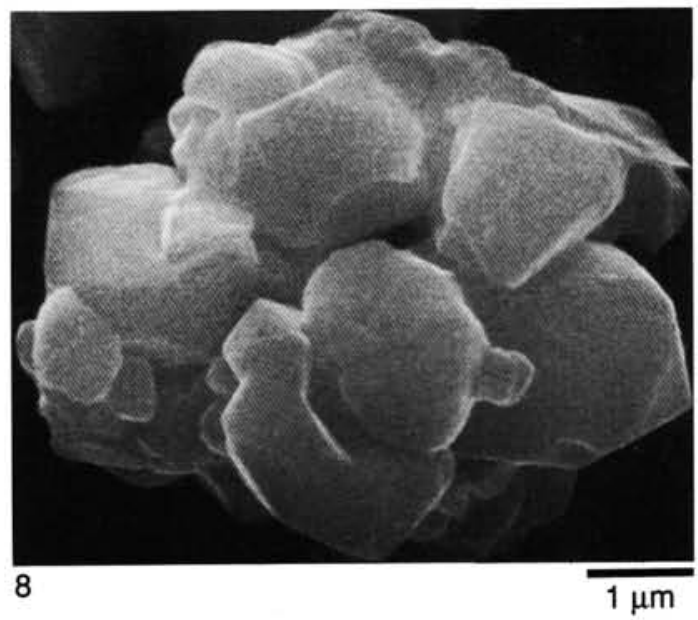

Plate 3. 1-2. Sphenolithus distentus, Sample 101-628A-25X-2, 80-81 cm. 3-5. Sphenolithus distentus, $\times 3300$, Sample 101-628A-25X-2, 80-81 $\mathrm{cm}$; (3) cross-polarized light, apical spine at $45^{\circ}$ to either polarizer; (4) transmitted light; (5) phase contrast. 6. Lanternithus minutus, showing dissolution, Section 101-628A-25X, CC. 7. Same specimen as 6, X3300, cross-polarized light. 8. Lanternithus minutus, showing dissolution, Section 101-628A-25X, CC. 9. Same specimen as $8, \times 3300$, cross-polarized light. 

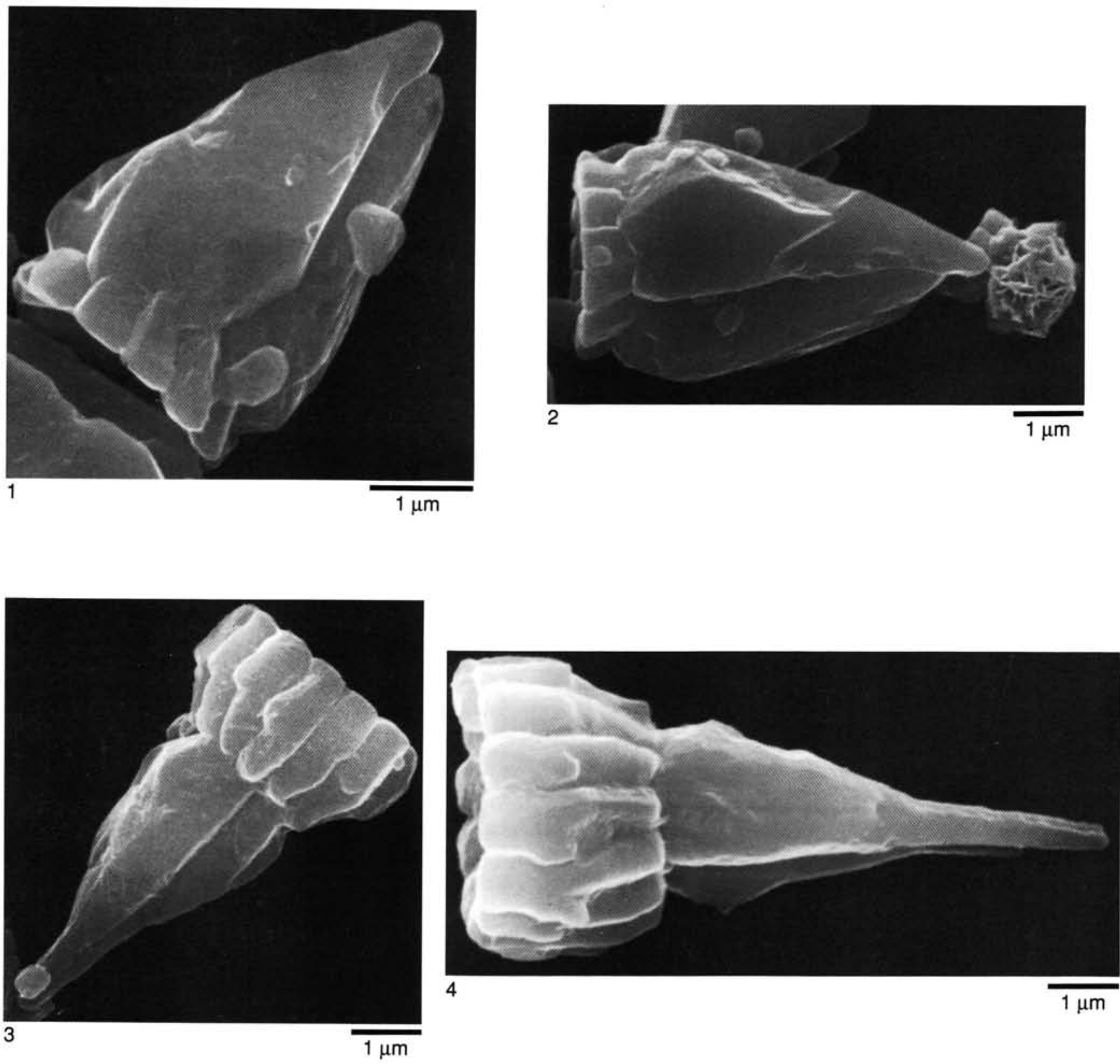

Plate 4. 1, 2. Sphenolithus predistentus, Section 101-628A-25X, CC. 3. Transitional form between Sphenolithus distentus and Sphenolithus ciperoensis, Sample 101-628A-21H-1, 80-81 cm. 4. Transitional form, Sample 101-628A-24X-1, 80-81 cm. 

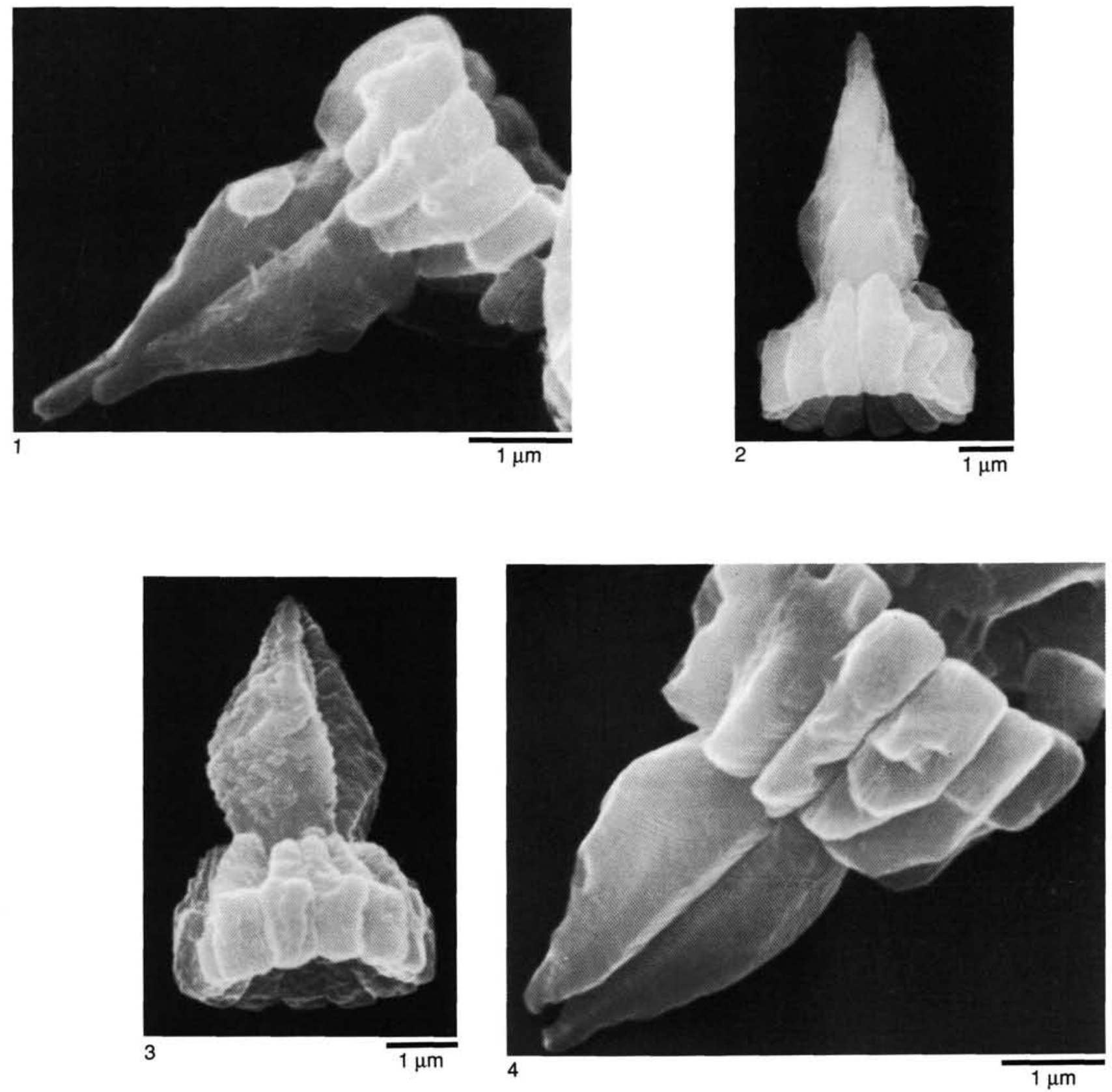

Plate 5. All specimens from Sample 101-628A-21H-1, 80-81 cm. 1, 4. Transitional form. 2. Transitional form showing slight dissolution. 3. Transitional form showing slight overgrowth. 

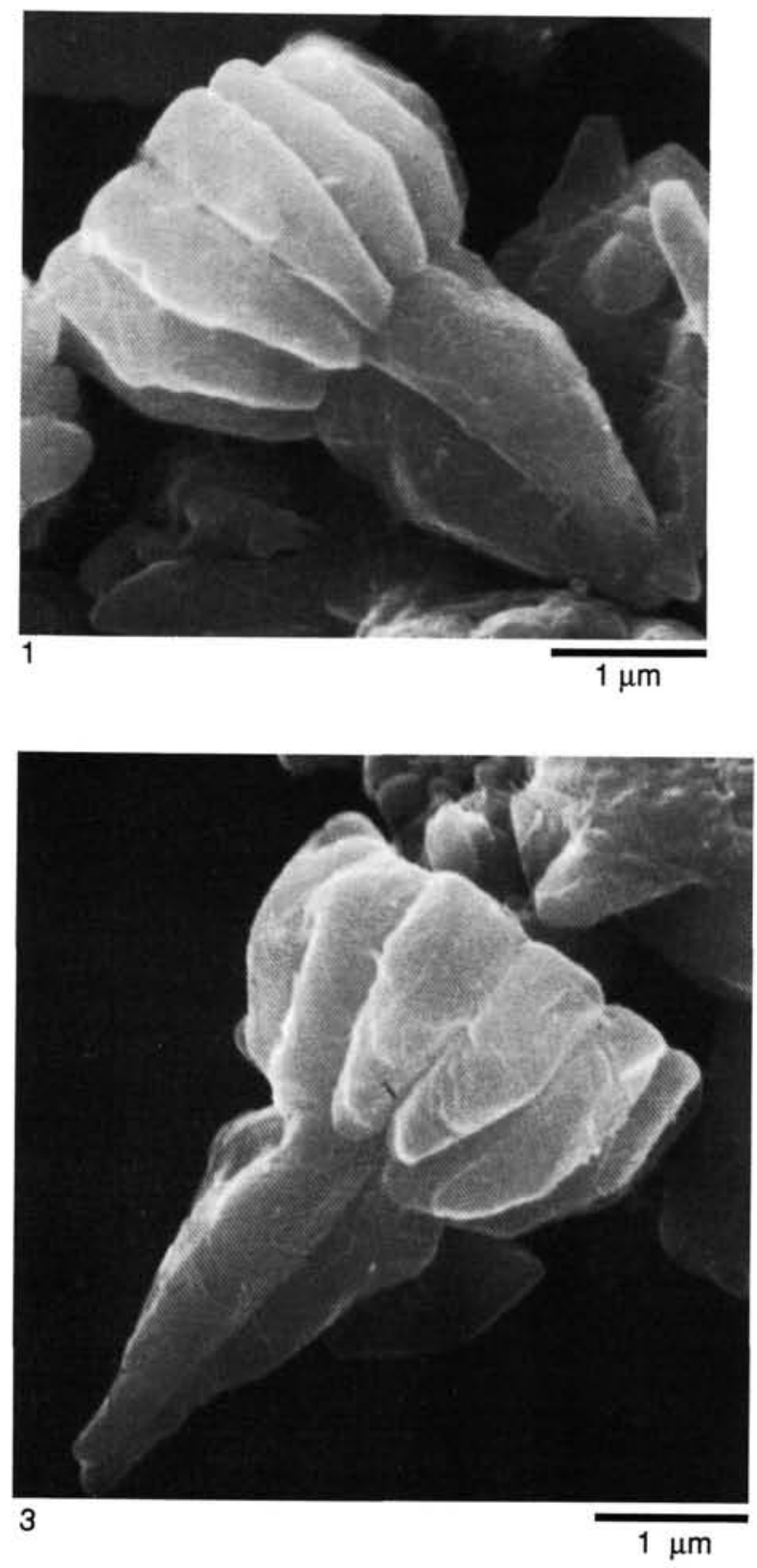

Plate 6. All specimens from Sample 101-628A-21H-1, 80-81 cm. 1-3. Sphenolithus ciperoensis. 4. Sphenolithus ciperoensis, showing slight dissolution.
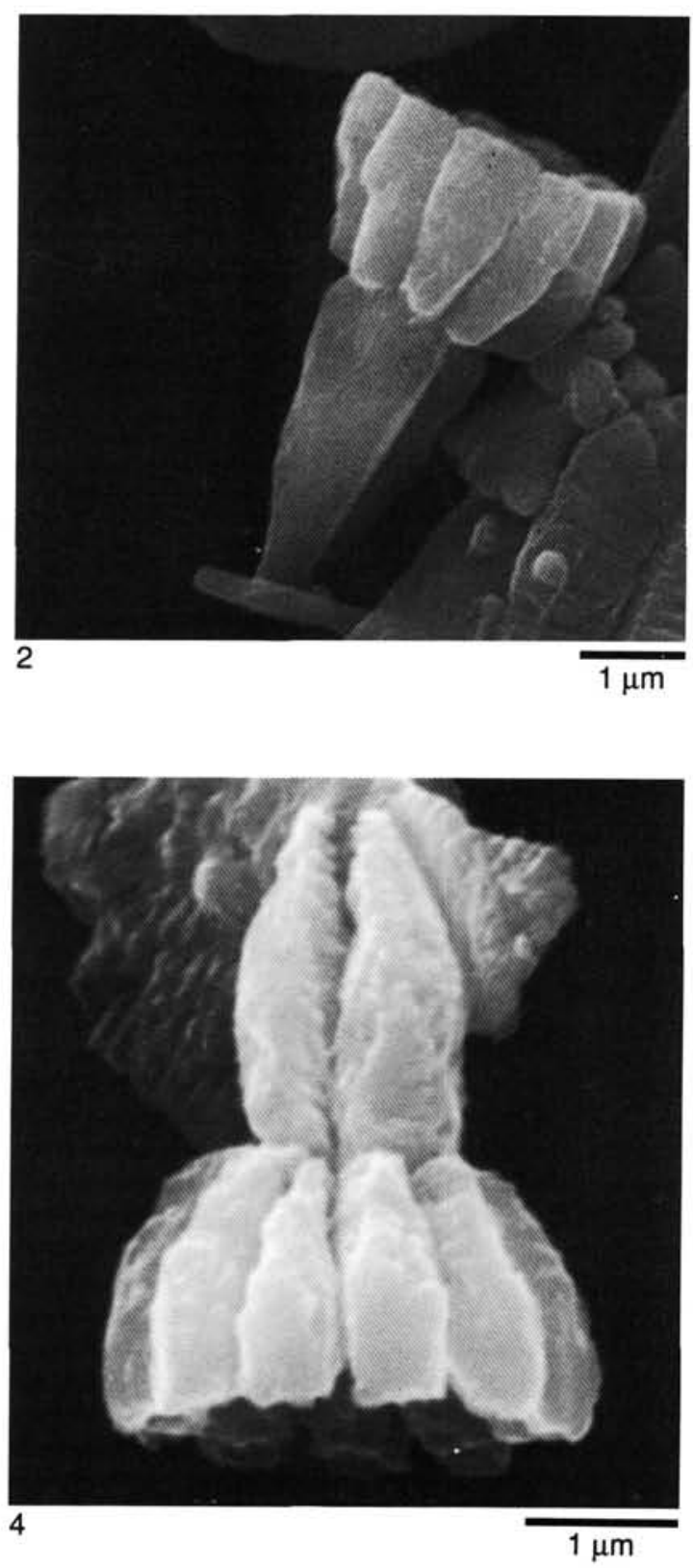

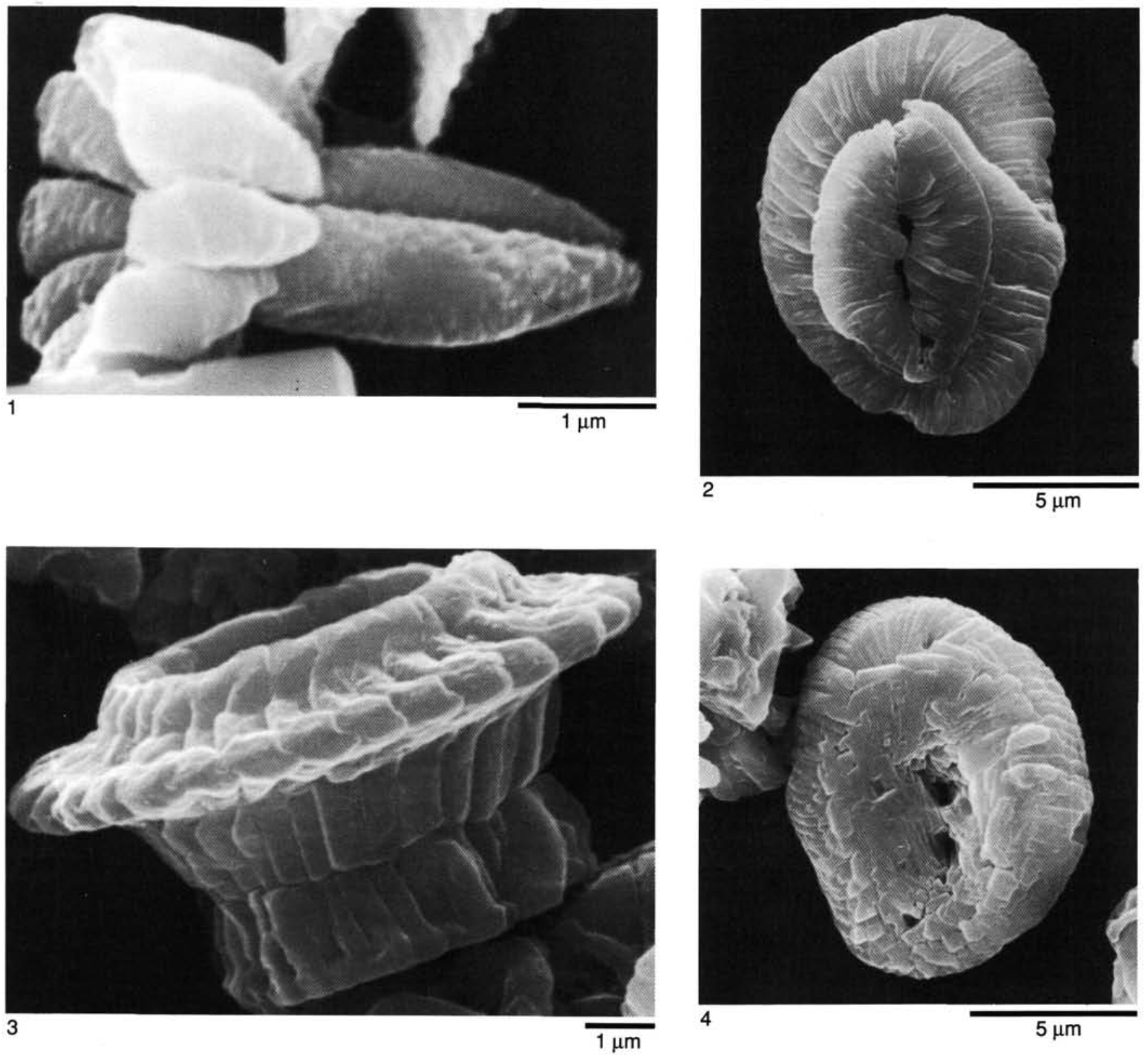

Plate 7. 1. Sphenolithus ciperoensis showing slight dissolution, Sample 101-628A-21H-1, 80-81 cm. 2, 4. Helicopontosphaera compacta, Sample 101-628A-29X-1, 80-81 cm; (2) proximal view; (4) distal view. 3. Hayella situliformis, Sample 101-628A-29X-1, 80-81 cm; lateral view. 